\title{
Standard Monomial Theory for Bott-Samelson Varieties
}

\author{
VENKATRAMANI LAKSHMIBAI, PETER LITTELMANN \\ and PETER MAGYAR \\ Department of Mathematics, Wells Hall, Michigan State University, \\ East Lansing, MI 48824, U.S.A.
}

(Received: 1 February 2000; accepted in final form: 6 November 2000)

\begin{abstract}
Bott-Samelson varieties are an important tool in geometric representation theory $[1,3,10,25]$. They were originally defined as desingularizations of Schubert varieties and share many of the properties of Schubert varieties. They have an action of a Borel subgroup, and the projective coordinate ring of a Bott-Samelson variety splits into certain generalized Demazure modules (which also appear in other contexts [22, 23]).

Standard Monomial Theory, developed by Seshadri and the first author [15, 16], and recently completed by the second author [20], gives explicit bases for the Demazure modules associated to Schubert varieties. In this paper, we extend the techniques of [20] to give explicit bases for the generalized Demazure modules associated to Bott-Samelson varieties, thus proving a strengthened form of the results announced by the first and third authors in [12] (see also [13]). We also obtain more elementary proofs of the cohomology vanishing theorems of Kumar [10] and Mathieu [25]; of the projective normality of Bott-Samelson varieties; and of the Demazure character formula.
\end{abstract}

Mathematic Subject Classification (2000). 14M15, 16G20, 14L30

Key words. Schubert variety, Young tableau, Demazure character formula, standard basis, vanishing theorem.

\section{Basis Theorem}

In this section we state the main results which we prove in the rest of the paper.

\subsection{DEMAZURE MODULES}

Let $G$ be a reductive algebraic group of rank $n$ over an algebraically closed field $\mathbf{k}, \mathbf{g}$ its Lie algebra, and $U(\mathbf{g})$ its universal enveloping algebra. (To avoid technicalities, we deal with finite-dimensional $\mathbf{g}$, but our results extend straightforwardly to symmetrizable Kac-Moody algebras.) Choose a Cartan subgroup $H \subset G$, and a Borel subgroup $B \supset H$. For $i=1, \ldots, n$, we then have positive and negative simple root vectors $E_{i}$ and $F_{i}$ generating $\mathbf{g}$; the Cartan subalgebra $\mathbf{h}$; the Borel subalgebra b generated by $E_{i}$ and $\mathbf{h}$; the simple roots $\alpha_{i}$ and coroots $\alpha_{i}^{\vee}=\left[E_{i}, F_{i}\right]$; the fundamental 
weights $\varpi_{i}$ with $\left\langle\varpi_{i}, \alpha_{j}^{\vee}\right\rangle=\delta_{i j}$ and the weight lattice $X=\bigoplus_{i=1}^{n} \mathbb{Z} \varpi_{i}$; and the simple reflections $s_{i}: X \rightarrow X, \lambda \mapsto \lambda-\left\langle\lambda, \alpha_{i}^{\vee}\right\rangle \alpha_{i}$, generating the Weyl group $W$.

An element $\lambda \in X$ is a one-dimensional representation $\lambda: \mathbf{h} \rightarrow \mathbf{k}$, and it extends to a one-dimensional $\mathbf{b}$-module denoted $\mathbf{k}_{\lambda}$. For a dominant weight $\lambda \in X^{+}:=\bigoplus_{i=1}^{n} \mathbb{Z}_{+} \varpi_{i}$, we let $V_{\lambda}$ denote the Weyl module of highest weight vector $v_{\lambda}$ with $b \cdot v_{\lambda}=\lambda(b) v_{\lambda}$ for $b \in \mathbf{b}$. (That is: over the rational numbers, $V_{\lambda, \mathbb{Q}}$ is the irreducible $G_{\mathbb{Q}}$-module; $V_{\lambda, \mathbb{Z}} \subset V_{\lambda, \mathbb{Q}}$ is the smallest $\mathbb{Z}$-submodule containing $v_{\lambda}$ and closed under the operations $E_{i}^{l} / l$ ! and $F_{i}^{l} / l$ ! for $l \geqslant 0, i \in[1, n]$; and in general $V_{\lambda}=V_{\lambda, \mathbf{k}}:=V_{\lambda, \mathbb{Z}} \otimes_{\mathbb{Z}} \mathbf{k}$.) We also have the dual module $V_{\lambda}^{*}$. For $\mathbf{k}$ of characteristic zero, $V_{\lambda}$ and $V_{\lambda}^{*}$ are irreducible $G$-modules.

Given an arbitrary word, meaning a sequence $\mathbf{i}=\left(i_{1}, \ldots, i_{r}\right)$ with $i_{j} \in\{1,2, \ldots, n\}$; as well as a multiplicity list $\mathbf{m}=\left(m_{1}, \ldots, m_{r}\right)$ with $m_{j} \in \mathbb{Z}_{+}$; we let

$$
\lambda_{1}:=m_{1} \varpi_{i_{1}}, \ldots, \lambda_{r}=m_{r} \varpi_{i_{r}} .
$$

We define the generalized Demazure module $V_{\mathbf{i}, \mathbf{m}}$ as a certain $B$-submodule of the tensor product $V_{\lambda_{1}} \otimes \cdots \otimes V_{\lambda_{r}}$ :

$$
V_{\mathbf{i}, \mathbf{m}}:=\mathbf{u}_{i_{1}} \cdot\left(v_{\lambda_{1}} \otimes \mathbf{u}_{i_{2}} \cdot\left(v_{\lambda_{2}} \otimes \cdots \otimes \mathbf{u}_{i_{r-1}} \cdot\left(v_{\lambda_{r-1}} \otimes \mathbf{u}_{i_{r}} \cdot\left(v_{\lambda_{r}}\right)\right) \cdots\right)\right),
$$

where $\mathbf{u}_{i}=\bigoplus_{l \geqslant 0} \mathbf{k} F_{i}^{l} / l$ ! denotes the hyperalgebra of a single negative root vector. (By convention, if $r=0$, so that $\mathbf{i}$ is the empty word, we set $V_{\mathbf{i}, \mathbf{m}}=\mathbf{k}_{0}$, the trivial one-dimensional $B$-module.) The dual $B$-module $V_{\mathbf{i}, \mathbf{m}}^{*}$ is a quotient of $V_{\lambda_{1}}^{*} \otimes \cdots \otimes V_{\lambda_{r}}^{*}$. We will explain in Section 1.4 how these modules arise from Bott-Samelson varieties.

The ordinary Demazure module $V_{\lambda}(w) \subset V_{\lambda}$ is essentially a special case. Given $w \in W$, choose the word $\mathbf{i}$ so that $w=s_{i_{1}} \cdots s_{i_{r}}$ is a reduced decomposition, and hence

$$
V_{\lambda}(w):=\mathbf{u}_{i_{1}} \cdots \mathbf{u}_{i_{r}} \cdot v_{\lambda} .
$$

Given a weight $\lambda=l_{1} \varpi_{1}+\cdots+l_{n} \varpi_{n}$, choose multiplicities $\left(m_{1}, \ldots, m_{r}\right)$ as follows. Suppose the rightmost occurrence of $i=1$ in the word $\mathbf{i}$ is at position $k$ : that is, $i_{k}=1, i_{j} \neq 1$ for $j>k$. Then let $m_{k}=l_{1}$. (If $i=1$ does not occur in $\mathbf{i}$, proceed to the next step.) Next let $k^{\prime}$ be the rightmost occurrence of $i=2$ in $\mathbf{i}$, and let $m_{k^{\prime}}=l_{2}$. Proceed in this way for each $i$, then let $m_{j}=0$ if it has not already been defined. Finally, let $\lambda^{\prime}=\sum_{i \notin i} l_{i} \varpi_{i}$, where the sum runs over those $i$ which do not occur anywhere in i. Then $V_{\lambda}(w) \cong \mathbf{k}_{\lambda^{\prime}} \otimes V_{\mathbf{i}, \mathbf{m}}$, with $w\left(\lambda^{\prime}\right)=\lambda^{\prime}$.

This paper revolves around the following problem.

PROBLEM. Find explicit bases for the generalized Demazure module $V_{\mathbf{i}, \mathbf{m}}$ and its dual $V_{i, \mathbf{m}}^{*}$.

From now on, we will assume $\mathbf{i}$ and $\mathbf{m}$ are fixed, so that we can refer to $i_{j}$, or $r$, or $\lambda_{1}, \ldots, \lambda_{r}$ without ambiguity. 


\subsection{STANDARD TABLEAUX}

We recall the machinery of Lakshmibai-Seshadri paths $([17,18])$ needed to index our bases of $V_{\mathbf{i}, \mathbf{m}}$.

Let $X_{\mathbb{R}}:=X \otimes_{\mathbb{Z}} \mathbb{R}$ be the real form of the weight lattice. A path is a piecewise-linear map $\pi:[0,1] \rightarrow X_{\mathbb{R}}$ (up to reparametrization) with $\pi(0)=0$. For a weight $\lambda \in X$, we let $\pi^{\lambda}$ denote the straight-line path: $\pi^{\lambda}(t):=t \lambda$; and $\pi_{1} * \pi_{2}$ denotes the concatenation of two paths. The weight of a path is its endpoint, $\mathrm{wt}(\pi):=\pi(1)$.

Let $W_{\lambda} \subset W$ be the stabilizer of a weight $\lambda \in X$, and use $>$ to denote the Chevalley-Bruhat order on $W$ and on the coset space $W / W_{\lambda}$. Let $\lambda \in X^{+}$be a dominant weight. An $L S$-chain of shape $\lambda$ is a pair of lists

$$
\left(\tau_{1}>\cdots>\tau_{q} ; 0=a_{0}<a_{1}<\cdots<a_{q}=1\right),
$$

where $\tau_{j} \in W / W_{\lambda}$ and $a_{j} \in \mathbb{Q}$, such that for each $j$ there exists a chain in the Bruhat order $\tau_{j}=\sigma_{0}>\sigma_{1}>\cdots>\sigma_{p}=\tau_{j+1} \quad$ with $\quad \ell\left(\sigma_{k+1}\right)=\ell\left(\sigma_{k}\right)+1 \quad$ and $a_{j}\left(\sigma_{k+1} \lambda-\sigma_{k} \lambda\right) \in \bigoplus_{i=1}^{n} \mathbb{Z} \alpha_{i}$ for each $k$. An LS-chain corresponds to a path $\pi:[0,1] \rightarrow X_{\mathbb{R}}$, whose linear pieces are defined by

$$
\pi(t)=\sum_{j=1}^{k-1}\left(a_{j}-a_{j-1}\right) \tau_{j} \lambda+\left(t-a_{k-1}\right) \tau_{k} \lambda \quad \text { for } \quad a_{k-1} \leqslant t \leqslant a_{k} .
$$

We call a path an $L S$-path if it can be so constructed from a (necessarily unique) LS-chain. We will frequently refer to LS-paths by their defining LS-chains, and abuse notation by writing: $\pi=\left(\tau_{1}>\cdots>\tau_{q} ; a_{0}<\cdots<a_{q}\right)$.

The lowering root operators $f_{i}$ act on a path $\pi$ in the usual way [18]. Since we will only consider $f_{i}$ acting on an LS-path or a concatenation of LS-paths, we may equivalently define these operators as in [17]. That is, let $Q$ be the minimum value of the function $t \mapsto\left\langle\pi(t), \alpha_{i}^{\vee}\right\rangle$ for $t \in[0,1]$. Let $t_{1}$ be the largest $t$ for which this minimum $Q$ is attained, and let $t_{2} \in[0,1]$ be the smallest $t>t_{1}$ where the function attains $Q+1$ (if there exists any such $t$ ). Now split our path $\pi$ into three segments $\pi=\pi_{1} * \pi_{2} * \pi_{3}$, corresponding to $t \in\left[0, t_{1}\right], t \in\left[t_{1}, t_{2}\right]$, and $t \in\left[t_{2}, 1\right]$. Define the root operator

$$
f_{i}(\pi):=\pi_{1} * s_{i} \pi_{2} * \pi_{3}
$$

where $s_{i} \pi_{2}$ is the path $t \mapsto s_{i}\left(\pi_{2}(t)\right)$. If there exists no $t_{2}$ as above, then $f_{i}(\pi)$ is undefined.

We may also define the raising root operators $e_{i}$ analogously, so that $e_{i}$ reflects the portion of $\pi$ between $t_{1}^{\prime}$ and $t_{2}^{\prime}$, where $t_{2}^{\prime}$ is the smallest $t$ for which $\left\langle\pi(t), \alpha_{i}^{\vee}\right\rangle$ attains its minimum $Q$, and $t_{1}^{\prime}$ is the largest $t<t_{2}^{\prime}$ where the function attains $Q+1$ (if there exists such $t)$. We thus have $e_{i}\left(f_{i}(\pi)\right)=\pi$ whenever $f_{i}(\pi)$ is defined, and $\operatorname{wt}\left(f_{i} \pi\right)=\operatorname{wt}(\pi)-\alpha_{i}, \operatorname{wt}\left(e_{i} \pi\right)=\operatorname{wt}(\pi)+\alpha_{i}$.

Recall our notation $\lambda_{1}=m_{1} \omega_{i_{1}}, \ldots, \lambda_{r}=m_{r} \omega_{i_{r}}$ for our fixed data $\mathbf{i}, \mathbf{m}$. A tableau or $L S$-monomial of shape $\left(\lambda_{1}, \ldots, \lambda_{r}\right)$ is a concatenation $\Pi=\pi_{1} * \cdots * \pi_{r}$, where $\pi_{j}$ is an 
LS-path of shape $\lambda_{j}$. A given tableau can be divided in only one way into such a concatenation of LS-paths of the proper shapes. Indeed, all LS-paths of shape $\lambda_{j}$ have length $\left|\lambda_{j}\right|$ (in the $W$-invariant metric); so if we divide the path $\Pi$ into pieces of length $\left|\lambda_{1}\right|, \ldots,\left|\lambda_{r}\right|$, and successively translate the division points to the origin, we obtain the unique LS-path factors $\pi_{1}, \ldots, \pi_{r}$.

We will usually refer to the tableau $\Pi$ by the corresponding $r$-tuple of LS-chains, and write: $\Pi=\left(\pi_{1}, \ldots, \pi_{r}\right)$. If we list all the Weyl group cosets in all the chains $\pi_{1}, \ldots, \pi_{r}$, we obtain a long list which we denote

$$
\bar{\Pi}=\left(\tau_{11}, \tau_{12}, \ldots, \tau_{1 p_{1}}, \tau_{21}, \ldots, \tau_{r p_{r}}\right),
$$

where $\tau_{j p}$ is a coset modulo $W_{\lambda_{j}}$ in the LS-chain $\pi_{j}$. When convenient we will reindex this long list as $\bar{\Pi}=\left(\tau_{1}, \tau_{2}, \ldots, \tau_{N}\right)$.

Denote by $[1, r]$ the set of integers $\{1,2, \ldots, r\}$. For any subset of indices $J=\{a<b<\cdots\} \subset[1, r]$, we have a subword $\mathbf{i}(J)=\left(i_{a}, i_{b}, \ldots\right)$ of our fixed word $\mathbf{i}=\left(i_{1}, \ldots, i_{r}\right)$. We also define $w(\mathbf{i})=s_{i_{1}} s_{i_{2}} \cdots s_{i_{r}} \in W$, so that $w(\mathbf{i}(J))=s_{i_{a}} s_{i_{b}} \cdots$, the Weyl group element corresponding to the subword $\mathbf{i}(J)$. We say that $\mathbf{i}$ is reduced if $r=\ell(w(\mathbf{i}))$, the Bruhat length; and similarly for the subword $\mathbf{i}(J)$. Further, we write $J^{(j)}:=J \cap[1, j]$, so that $w\left(\mathbf{i}\left(J^{(j)}\right)\right)$ is an initial subword of $w(\mathbf{i}(J))$. If $\mathbf{i}(J)$ is reduced, then so is $\mathbf{i}\left(J^{(j)}\right)$.

Let $\Pi$ be a tableau of shape $\left(\lambda_{1}, \ldots, \lambda_{r}\right)$, considered as a sequence $\left(\pi_{1}, \ldots, \pi_{r}\right)$ of LS-chains $\pi_{j}$, producing the long list of cosets $\bar{\Pi}=\left(\tau_{11}, \ldots, \tau_{r p_{r}}\right)$.

DEFINITION. We say $\Pi$ is a liftable-standard tableau (or just liftable) if there exists a long chain of position-sets $\left(J_{11} \supset \cdots \supset J_{r p_{r}}\right)$ such that for all $j, p$, the subword $\mathbf{i}\left(J_{j p}^{(j)}\right)$ is reduced and

$$
w\left(\mathbf{i}\left(J_{j p}^{(j)}\right)\right) \equiv \tau_{j p} \text { modulo } W_{\lambda_{j}} .
$$

Now consider the tableau $\Pi$ as a concatenation of LS-paths $\pi_{1} * \cdots * \pi_{r}$.

DEFINITION. We say a tableau $\Pi$ is a constructable-standard tableau (or is constructable) if it can be written as

$$
\Pi=f_{i_{1}}^{l_{1}}\left(\pi^{\lambda_{1}} * f_{i_{2}}^{l_{2}}\left(\pi^{\lambda_{2}} * \cdots f_{i_{r}}^{l_{r}}\left(\pi^{\lambda_{r}}\right) \cdots\right)\right)
$$

for some $l_{1}, \ldots, l_{r} \in \mathbb{Z}_{+}$.

Note that for any $l_{1}, \ldots, l_{r} \in \mathbb{Z}_{+}$, the path $\Pi$ defined by the previous formula is a concatenation of LS-paths of the correct shapes, and is thus a tableau, which is constructable-standard by the definition. (See [18, $\S 2.6$ and 4.2].)

Given an arbitrary path $\Pi$ of shape $\left(\lambda_{1}, \ldots, \lambda_{r}\right)$, we may test whether it is a standard tableau as follows. Define the highest raising $e^{\text {top }}(\Pi):=e^{l}(\Pi)$, where $l$ is maximal such that $e^{l}(\Pi)$ is defined. Let $e_{i_{1}}^{\text {top }}(\Pi)=\pi_{1}^{\prime} * \cdots * \pi_{r}^{\prime}$ (division into pieces of length $\left.\left|\lambda_{1}\right|, \ldots,\left|\lambda_{r}\right|\right)$. If $\pi_{1}^{\prime} \neq \pi^{\lambda_{1}}$, then $\Pi$ is not standard. Otherwise, let 
$\Pi^{\prime}=\pi_{2}^{\prime} * \cdots * \pi_{r}^{\prime}$ and let $e_{i_{2}}^{\text {top }}\left(\Pi^{\prime}\right)=\pi_{2}^{\prime \prime} * \cdots * \pi_{r}^{\prime \prime}$. If $\pi_{2}^{\prime \prime} \neq \pi^{\lambda_{2}}$, then $\Pi$ is not standard. If we can continue in this way, raising by $e_{i_{j}}$ and removing intial factors $\pi^{\lambda_{j}}$, until we obtain the one-point path (of length 0 ), then $\Pi$ is standard; otherwise it is not.

EXAMPLE. Let $G=G L_{3}, \mathbf{i}=(1,2,1,2)$ a non-reduced word, $\mathbf{m}=(2,1,0,1)$, $X=\mathbb{Z}_{\varepsilon_{1}} \oplus \mathbb{Z} \varepsilon_{2}, \varpi_{1}=\varepsilon_{1}, \varpi_{2}=\varepsilon_{1}+\varepsilon_{2}, \lambda_{1}=2 \varepsilon_{1}, \lambda_{2}=\varepsilon_{1}+\varepsilon_{2}, \lambda_{3}=0, \lambda_{4}=\varepsilon_{1}+\varepsilon_{2}$.

A typical LS-path of shape $\lambda_{1}$ is: $\pi=\left(s_{1}>e ; 0<\frac{1}{2}<1\right)=\pi^{\varepsilon(2)} * \pi^{\varepsilon(1)}$, a concatenation of two length-one segments. (For legibility we write $\varepsilon(1), \varepsilon(2)$ instead of $\varepsilon_{1}, \varepsilon_{2}$; and $\pi^{\mu}$ denotes a straight-line path.)

Now consider the tableau $\Pi=\pi^{\varepsilon(2)} * \pi^{\varepsilon(1)} * \pi^{\varepsilon(1)+\varepsilon(3)} * \pi^{\varepsilon(2)+\varepsilon(3)}$. Dividing $\Pi$ into segments of lengths $2,1,0,1$, we get the LS-path factors of shapes $\lambda_{1}, \ldots, \lambda_{4}$ : $\Pi=\pi_{1} * \pi_{2} * \pi_{3} * \pi_{4}$, where:

$$
\begin{aligned}
& \pi_{1}=\pi^{\varepsilon(2)} * \pi^{\varepsilon(1)}=\left(s_{1}>e ; 0<\frac{1}{2}<1\right), \\
& \pi_{2}=\pi^{\varepsilon(1)+\varepsilon(3)}=\left(s_{2} ; 0<1\right), \\
& \pi_{3}=\pi^{0}=(e ; 0<1), \text { the one }- \text { point path, } \\
& \pi_{4}=\pi^{\varepsilon(2)+\varepsilon(3)}=\left(s_{2} s_{1} ; 0<1\right) .
\end{aligned}
$$

This path has the lifting: $J_{11}=\{1,2,3,4\}, J_{12}=\{2,3,4\}, J_{21}=\{2,3,4\}, J_{31}=\{3,4\}$, $J_{41}=\{3,4\}$; since:

$$
\begin{aligned}
& w\left(\mathbf{i}\left(J_{11}^{(1)}\right)\right)=w\left(i_{1}\right)=s_{1} \equiv \tau_{11} W_{\lambda_{1}}, \\
& w\left(\mathbf{i}\left(J_{12}^{(1)}\right)\right)=w(\emptyset)=e \equiv \tau_{12} W_{\lambda_{1}}, \\
& w\left(\mathbf{i}\left(J_{21}^{(2)}\right)\right)=w\left(i_{2}\right)=s_{2} \equiv \tau_{21} W_{\lambda_{2}}, \\
& w\left(\mathbf{i}\left(J_{31}^{(3)}\right)\right)=w\left(i_{3}\right)=s_{1} \equiv e \equiv \tau_{31} W_{\lambda_{3}}, \\
& w\left(\mathbf{i}\left(J_{41}^{(4)}\right)\right)=w\left(i_{3} i_{4}\right)=s_{1} s_{2} \equiv \tau_{41} W_{\lambda_{4}} .
\end{aligned}
$$

Furthermore, $\Pi$ is constructable, since:

$$
\begin{aligned}
\Pi & =f_{1}\left(\pi^{2 \varepsilon(1)} * f_{2}\left(\pi^{\varepsilon(1)+\varepsilon(2)} * f_{1} f_{2}\left(\pi^{\varepsilon(1)+\varepsilon(2)}\right)\right)\right) \\
& =f_{1}\left(\pi^{\lambda_{1}} * f_{2}\left(\pi^{\lambda_{2}} * f_{1}\left(\pi^{\lambda_{3}} * f_{2}\left(\pi^{\lambda_{4}}\right)\right)\right)\right)
\end{aligned}
$$

THEOREM 1. A tableau $\Pi$ is liftable if and only if it is constructable. We call such $\Pi$ $a$ standard tableau of shape $\left(\lambda_{1}, \ldots, \lambda_{r}\right)$.

Any set $\mathcal{T}$ of paths possesses a natural structure of crystal graph: namely, the graph with vertex set $\mathcal{T}$, and with $i$-colored edges $\left\{\Pi, f_{i} \Pi\right\}$ (whenever both $\Pi$ and $f_{i} \Pi$ lie in $\mathcal{T}$ ). For example, the crystal graph of an ordinary Demazure module $V_{\lambda}(w)$ is associated to the set of tableaux $\left\{f_{i_{1}}^{l_{1}} \ldots f_{i_{r}}^{l_{r}} \pi^{\lambda} \mid l_{1}, \ldots, l_{r} \geqslant 0\right\}$, where $w=s_{i_{1}} \cdots s_{i_{r}}$ (reduced). Many important crystal graphs reduce to this basic case. 
THEOREM 2. The crystal graph on the set $\{\Pi\}$ of standard tableaux of shape $\left(\lambda_{1}, \ldots, \lambda_{r}\right)$ is isomorphic (as an edge-colored graph) to a disjoint union of crystal graphs of ordinary Demazure modules. For $\mu \in X^{+}$a dominant weight, the same is true of the set of paths $\left\{\pi^{\mu} * \Pi\right\}$, where $\Pi$ runs over the standard tableaux of shape $\left(\lambda_{1}, \ldots, \lambda_{r}\right)$.

This is a combinatorial version of excellent filtration for the $B$-modules $V_{\mathbf{i}, \mathbf{m}}^{*}$ and $\mathbf{k}_{\mu}^{*} \otimes V_{\mathbf{i}, \mathbf{m}}^{*}$. (See Section 2.4.)

\subsection{STANDARD MONOMIAL BASES}

We use tableaux to index bases of $B$-modules, starting with the Weyl modules $V_{\lambda}$, then proceeding to $V_{\mathbf{i}, \mathbf{m}}$.

For any Weyl module $V_{\lambda}$, the second author has constructed [20] a basis $\left\{v_{\pi}\right\}$ indexed by LS-chains $\pi$ of shape $\lambda$, in which $v_{\pi}$ is a weight vector with weight $\operatorname{wt}(\pi)$. (See Section 3.1 below for details.) The basis $\left\{v_{\pi}\right\}$, inspired by the work of Raghavan and Sankaran [26], is highly non-canonical, depending on several arbitrary choices. However $\left\{v_{\pi}\right\}$ is related to most 'reasonable' bases of $V_{\lambda}$ by a triangular matrix. Actually, we shall find it more convenient to pair $\left\{v_{\lambda}\right\}$ with bases of the dual module $V_{\lambda}^{*}$.

To be more specific, define the following lexicographic partial order on LS-chains. Given $\pi=\left(\tau_{1}>\cdots ; 0<a_{1}<\cdots\right)$ and $\theta=\left(\sigma_{1}>\cdots ; 0<b_{1}<\cdots\right)$, we say $\pi<\theta$ if $\tau_{1}<\sigma_{1}$ (in Bruhat order); or $\tau_{1}=\sigma_{1}, a_{1}<b_{1}$; or $\tau_{1}=\sigma_{1}, a_{1}=b_{1}, \tau_{2}<\sigma_{2}$; etc. Note that the highest weight path is minimal in this order, and a path is large in this order if it is far from the highest weight path. We can extend this to tableaux by defining $\left(\pi_{1}, \ldots, \pi_{r}\right)<\left(\theta_{1}, \ldots, \theta_{r}\right)$ to mean: $\pi_{1}<\theta_{1}$ (in the above order); or $\pi_{1}=\theta_{1}$ and $\pi_{2}<\theta_{2}$; etc.

Given a basis $\left\{p_{\pi}\right\}$ of $V_{\lambda}^{*}$ indexed by LS-chains of shape $\lambda$, we say $\left\{p_{\pi}\right\}$ is triangular to $\left\{v_{\pi}\right\}$ if

$$
p_{\pi}=v_{\pi}^{*}+\sum_{\theta>\pi} \propto v_{\theta}^{*}
$$

where $\propto$ indicates an appropriate scalar coefficient (possibly different in each term). That is, $\left\langle p_{\pi}, v_{\pi}\right\rangle=1$, and $\left\langle p_{\theta}, v_{\pi}\right\rangle=0$ for all $\theta \neq \pi$. A certain basis $\left\{p_{\pi}\right\}$ defined in [20] obeys this triangular property for all groups $G$, as do most of the other known bases of Weyl modules, at least for classical groups $G$ (types $A_{n}, B_{n}, C_{n}, D_{n}$ ).

THEOREM 3. The following bases of $V_{\lambda}^{*}$ are triangular to $\left\{v_{\pi}\right\}$, for the specified classes of reductive groups:

(a) Littelmann's canonically defined LS-path basis [20], for all G;

(b) Lusztig's dual canonical basis [21] (= Kashiwara's upper crystal basis [6, 8]), for classical $G$; 
(c) Lakshmibai's standard PBW basis [11] for classical G.

(d) Lakshmibai-Seshadri's standard monomial basis [15, 16], for classical G.

Proof. For (a), see [20]. It is established in [24, 26] that, for the fundamental representations $V_{W_{i}}^{*}$, the bases (a), (b), and (d) coincide. But this implies the triangularity for an arbitrary $V_{\lambda}^{*}$. The triangularity between (b) and (c) follows from [11].

We expect that bases (b)-(d) possess the triangularity property for all $G$ (including the Kac-Moody case).

Any such system of bases $\left\{p_{\pi}\right\}$ for each $V_{\lambda}^{*}$ gives a basis of $V_{\lambda_{1}}^{*} \otimes \cdots \otimes V_{\lambda_{r}}^{*}$ whose indexing set consists of all tableaux of shape $\left(\lambda_{1}, \ldots, \lambda_{r}\right)$. The standard tableaux pick out a subset of this basis which restricts to a basis of the quotient $V_{\mathbf{i}, \mathbf{m}}^{*}$.

THEOREM 4. For every $\lambda \in X^{+}$, let $\left\{p_{\pi}\right\}$ be a basis of $V_{\lambda}^{*}$ which is triangular to $\left\{v_{\pi}\right\}$. For $\Pi=\left(\pi_{1}, \ldots, \pi_{r}\right)$ a tableau of shape $\left(\lambda_{1}, \ldots, \lambda_{r}\right)$, define

$$
p_{\Pi}:=p_{\pi_{1}} \otimes \cdots \otimes p_{\pi_{r}} \in V_{\lambda_{1}}^{*} \otimes \cdots \otimes V_{\lambda_{r}}^{*} .
$$

Then $\left\{p_{\Pi}\right\}$, where $\Pi$ runs over the standard tableau of shape $\left(\lambda_{1}, \ldots, \lambda_{r}\right)$, restricts to a basis of $V_{\mathbf{i}, \mathbf{m}}^{*}$. We call this a standard monomial basis of $V_{\mathbf{i}, \mathbf{m}}^{*}$.

Since we may assume $p_{\Pi}$ to have weight $\mathrm{wt}(\Pi)$, we can use the combinatorics of tableaux to compute the character of $V_{\mathbf{i}, \mathbf{m}}$. Let $R=\mathbb{Z}[X]=\bigoplus_{\lambda \in X} \mathbb{Z} \mathrm{e}^{\lambda}$ be the group ring of the weight lattice $X$. The Weyl group acts $\mathbb{Z}$-linearly on characters by $w\left(\mathrm{e}^{\lambda}\right)=\mathrm{e}^{w(\lambda)}$. We may define the Demazure operator $\Lambda_{i}: R \rightarrow R$ by

$$
\Lambda_{i}(f):=\frac{f-\mathrm{e}^{-\alpha_{i}} s_{i}(f)}{1-\mathrm{e}^{-\alpha_{i}}}
$$

which can be interpreted uniquely as an element of $R$. We may also characterize $\Lambda_{i}$ as the unique linear operator with $\Lambda_{i}^{2}=\Lambda_{i}$ and

$$
\Lambda_{i}\left(\mathrm{e}^{\lambda}\right)=\mathrm{e}^{\lambda}+\mathrm{e}^{\lambda-\alpha_{i}}+\mathrm{e}^{\lambda-2 \alpha_{i}}+\cdots+\mathrm{e}^{s_{i} \lambda} .
$$

for any $\lambda \in X$ with $\left\langle\lambda, \alpha_{i}^{\vee}\right\rangle \geqslant 0$.

THEOREM 5. The character of the B-module $V_{\mathbf{i}, \mathbf{m}}$ is

$$
\Lambda_{i_{1}}\left(\mathrm{e}^{\lambda_{1}} \Lambda_{i_{2}}\left(\mathrm{e}^{\lambda_{2}} \cdots \Lambda_{i_{r}}\left(\mathrm{e}^{\lambda_{r}}\right) \cdots\right)\right)
$$

Our strategy of proof for the above theorems is as follows. Theorem 1 is an elementary combinatorial fact, proved in Section 2.2. Also in Section 2.3 we prove that the Demazure formula computes the formal character of the set of standard tableaux, and we prove Theorem 2 in Section 2.4, all by the combinatorics of paths. Next we prove Theorem 4 in two steps. First, in Section 3 we show that the set 
$\left\{p_{\Pi}\right\}$ is linearly independent in $V_{\mathbf{i}, \mathbf{m}}^{*}$, so that

$\operatorname{dim} V_{\mathbf{i}, \mathbf{m}}^{*} \geqslant \operatorname{dim} \operatorname{Span}\left\langle p_{\Pi}\right\rangle=\#$ standard tableaux.

Then in Section 4 we compare the combinatorial Demazure formula with the geometric version to conclude

\# standard tableaux $\geqslant \operatorname{dim} V_{\mathbf{i}, \mathbf{m}}^{*}$,

which proves the Theorem. Also, Theorem 5 follows in the course of this proof, as does Theorem 6 below.

\subsection{BOTT-SAMELSON VARIETIES}

We now give a Borel-Weil-type result for producing our Demazure modules inside the projective coordinate rings of certain varieties. We will prove this, along with the corresponding analog of Bott's vanishing theorem. (These theorems were originally proved in our case in [25] and [10].)

Recall our fixed word $\mathbf{i}=\left(i_{1}, \ldots, i_{r}\right)$ from Section 1.1, and our Borel subgroup $B$ of $G$. For each $i$ let $P_{i} \supset B$ be the minimal parabolic subgroup with Weyl group $\left\langle s_{i}\right\rangle$, and define the Bott-Samelson variety as the quotient

$$
Z_{\mathbf{i}}:=\left(P_{i_{1}} \times \cdots \times P_{i_{r}}\right) / B^{r},
$$

where $B^{r}$ acts on the right by:

$$
\left(p_{1}, \ldots, p_{r}\right) \cdot\left(b_{1}, \ldots, b_{r}\right):=\left(p_{1} b_{1}, b_{1}^{-1} p_{2} b_{2}, \ldots, b_{r-1}^{-1} p_{r} b_{r}\right) .
$$

This is a smooth algebraic variety of dimension $r$. (If $r=0$ and $\mathbf{i}$ is the empty word, we let $Z_{\mathbf{i}}$ be a point.)

For $\lambda \in X$, let $\mathrm{e}^{\lambda}$ denote the multiplicative character of $B$ associated to $\lambda$, and let $\mathbf{k}_{\left(\lambda_{1}, \ldots, \lambda_{r}\right)}^{*}$ be the one-dimensional representation of $B^{r}$ defined by

$$
\left(b_{1}, \ldots, b_{r}\right)^{-1} \cdot k:=\mathrm{e}^{\lambda_{1}}\left(b_{1}\right) \cdots \mathrm{e}^{\lambda_{r}}\left(b_{r}\right) k .
$$

Define a line bundle on $Z_{\mathbf{i}}$ by

$$
\mathcal{L}_{\mathbf{i}, \mathbf{m}}:=\left(P_{i_{1}} \times \cdots \times P_{i_{r}}\right) \stackrel{B^{r}}{\times} \mathbf{k}_{\left(\lambda_{1}, \ldots, \lambda_{r}\right)}^{*}
$$

so that we identify

$$
(\tilde{p}, k) \sim\left(\tilde{p} \cdot \tilde{b}, \tilde{b}^{-1} \cdot k\right)
$$

for $\tilde{p} \in P_{i_{1}} \times \cdots \times P_{i_{r}}, \quad \tilde{b} \in B^{r}$, and $k \in \mathbf{k}$. Unraveling the definitions, we may 
concretely describe the space of regular global sections of this bundle as:

$$
H^{0}\left(Z_{\mathbf{i}}, \mathcal{L}_{\mathbf{i}, \mathbf{m}}\right)=\left\{\begin{array}{l|c}
f: P_{i_{1}} \times \cdots \times P_{i_{r}} \rightarrow \mathbf{k} & \begin{array}{c}
\forall b_{j} \in B, p_{j} \in P_{i_{j}} \\
f\left(\left(p_{1}, \ldots, p_{r}\right) \cdot\left(b_{1}, \ldots, b_{r}\right)\right)= \\
\mathrm{e}^{\lambda_{1}}\left(b_{1}\right) \ldots \mathrm{e}^{\lambda_{r}}\left(b_{r}\right) f\left(p_{1}, \ldots, p_{r}\right)
\end{array}
\end{array}\right\},
$$

where $f$ denotes a polynomial function on the linear algebraic group $P_{i_{1}} \times \cdots \times P_{i_{r}}$.

The Borel subgroup acts on $Z_{\mathbf{i}}$ and $\mathcal{L}_{\mathbf{i}, \mathbf{m}}$ by left multiplication: for $b \in B$,

$$
b \cdot\left(p_{1}, \ldots, p_{r}\right):=\left(b p_{1}, p_{2}, \ldots, p_{r}\right) \text { and } b \cdot\left(p_{1}, \ldots, p_{r}, k\right):=\left(b p_{1}, p_{2}, \ldots, p_{r}, k\right) .
$$

The space $H^{0}\left(Z_{\mathbf{i}}, \mathcal{L}_{\mathbf{i}, \mathbf{m}}\right)$ of regular global sections of $\mathcal{L}_{\mathbf{i}, \mathbf{m}}$ over $Z_{\mathbf{i}}$ is naturally a $B$-module under translation.

Our analysis extends to certain varieties desingularized by Bott-Samelson varieties, called configuration varieties in $[22,23]$. For a given $\mathbf{m}=\left(m_{1}, \ldots, m_{r}\right)$, the line bundle $\mathcal{L}_{\mathbf{i}, \mathbf{m}}$ is ample (resp. semi-ample) when all $m_{j}>0$ (resp. $m_{j} \geqslant 0$ ). In the latter case, define $Z_{\mathbf{i}, \mathbf{m}}$ as the image of the natural map $Z_{\mathbf{i}} \rightarrow \mathbb{P}^{*} H^{0}\left(Z_{\mathbf{i}}, \mathcal{L}_{\mathbf{i}, \mathbf{m}}\right)$. This variety is singular in general, and can be of smaller dimension than $Z_{\mathbf{i}}$. If we take $\mathbf{i}, \mathbf{m}$ so that $\mathbf{k}_{\lambda^{\prime}} \otimes V_{\mathbf{i}, \mathbf{m}} \cong V_{\lambda}(w)$ is a Demazure module (see Section 1.1), then $Z_{\mathbf{i}, \mathbf{m}} \cong \overline{B \cdot w B} \subset G / B$, a Schubert variety.

THEOREM 6. The B-module of regular global sections is isomorphic to the dual of a generalized Demazure module:

$$
H^{0}\left(Z_{\mathbf{i}}, \mathcal{L}_{\mathbf{i}, \mathbf{m}}\right) \cong V_{\mathbf{i}, \mathbf{m}}^{*}
$$

Also, $Z_{\mathbf{i}}$ is projectively normal with respect to the bundle $\mathcal{L}_{\mathbf{i}, \mathbf{m}}$, and $H^{i}\left(Z_{\mathbf{i}}, \mathcal{L}_{\mathbf{i}, \mathbf{m}}\right)=0$ for $i \geqslant 1$. Furthermore, all the above statements hold for $Z_{\mathbf{i}, \mathbf{m}}$ in place of $Z_{\mathbf{i}}$.

We give the proof in Section 4.2.

\subsection{THE SYMPLECTIC GROUP}

In [13], we work out the above constructions at length in the case of the general linear group $G=G L_{n+1}$. (Our treatment there is more elementary, avoiding the technicalities of the basis $\left\{v_{\Pi}\right\}$.) In this section, we give the example of the symplectic group $G=S p_{2 n}$, in the spirit of De Concini [2]. (The orthogonal case is similar, but slightly more complicated.)

In general, the main obscurity in the above constructions is that most bases $\left\{p_{\pi}\right\}$ of $V_{\lambda}^{*}$ are difficult to write explicitly, so that writing the corresponding basis $\left\{p_{\Pi}\right\}$ of $V_{\mathbf{i}, \mathbf{m}}^{*}$ is equally difficult. However for $G$ a classical group, it is easier to construct bases of the fundamental representations $V_{W_{i}}^{*}$, and to obtain from these a standard monomial basis $\left\{p_{\pi}\right\}$ of the quotient $\left(V_{w_{i}}^{*}\right)^{\otimes m} \rightarrow V_{m \varpi_{i}}^{*}=V_{\lambda_{i}}^{*}$. (See $[15,16]$.) Thus we will obtain 
bases of $V_{\mathbf{i}, \mathbf{m}}^{*}$ via the composite restriction map:

$$
\rho:\left(V_{\varpi_{i_{1}}}^{*}\right)^{\otimes m_{1}} \otimes \cdots \otimes\left(V_{\varpi_{i_{r}}}^{*}\right)^{\otimes m_{r}} \rightarrow V_{\lambda_{1}}^{*} \otimes \cdots \otimes V_{\lambda_{r}}^{*} \rightarrow V_{\mathbf{i}, \mathbf{m}}^{*}
$$

This is the formulation announced in [12].

Now, for $i \in[1,2 n]$, let us denote $\bar{i}:=2 n+1-i$ and $|i|:=\min (i, \bar{i})$. The standard basis of $\mathbf{k}^{2 n}$ is $\left\{e_{1}, \ldots, e_{2 n}\right\}=\left\{e_{1}, \ldots, e_{n}, e_{\bar{n}}, \ldots, e_{\overline{1}}\right\}$. Let $G=S p_{2 n}(\mathbf{k})$ be the linear isometries of the symplectic form $\left\langle e_{i}, e_{\bar{j}}\right\rangle=-\left\langle e_{\bar{j}}, e_{i}\right\rangle=\delta_{i j},\left\langle e_{i}, e_{j}\right\rangle=\left\langle e_{i}, e_{\bar{j}}\right\rangle=0$ for $i, j \in[1, n]$. That is, $G=\left\{A \in G L_{2 n} \mid A E A^{t}=E\right\}$, where $E$ is the matrix with $i j$-coordinate $\left\langle e_{i}, e_{j}\right\rangle$ for $i, j \in[1,2 n]$.

We may write the weight lattice as $X=\bigoplus_{i=1}^{n} \mathbb{Z} \varepsilon_{i}$; with simple roots $\alpha_{1}=\varepsilon_{1}-\varepsilon_{2}, \ldots, \alpha_{n-1}=\varepsilon_{n-1}-\varepsilon_{n}, \quad \alpha_{n}=2 \varepsilon_{n} ; \quad$ fundamental weights $\varpi_{i}=\varepsilon_{1}+\varepsilon_{2}+\cdots+\varepsilon_{i} ;$ and simple coroots $\alpha_{1}^{\vee}=\varepsilon_{1}^{*}-\varepsilon_{2}^{*}, \ldots, \alpha_{n-1}^{\vee}=\varepsilon_{n-1}^{*}-\varepsilon_{n}^{*}$, $\alpha_{n}^{\vee}=\varepsilon_{n}^{*}$. An element of the Weyl group $W$ may be indexed by a signed permutation: a map $w:[1, n] \rightarrow[1,2 n]=\{1, \ldots, n, \bar{n}, \ldots, \overline{1}\}$ such that $|w|: i \mapsto|w(i)|$ is a permutation of $[1, n]$. Such a $w$ acts on $X$ by $w\left(\varepsilon_{i}\right):=\varepsilon_{w(i)}$, where we write $\varepsilon_{\bar{i}}:=-\varepsilon_{i}$ for $i \in[1, n]$.

We can realize the fundamental representations $V_{\varpi_{i}}^{*}$ inside the coordinate $\operatorname{ring} \mathbf{k}[G]$ of polynomial functions on the affine variety $G$. (The group acts on functions via left translation: $(g \cdot f)(A):=f\left(g^{-1} A\right)$ for $f \in \mathbf{k}[G], g, A \in G$.) That is, we have

$$
V_{\varpi_{i}}^{*} \cong \operatorname{Span}\left\langle p_{\tau} \mid \tau \subset[1,2 n], \# \tau=i\right\rangle,
$$

where $p_{\tau}=p_{\tau}(A)$ is the minor of the matrix $A$ on the first $i$ columns and on the rows $\tau$.

To use the above model, it is most convenient to index the basis of the fundamental-weight modules $V_{\Phi_{i}}$ not by LS-paths, but by certain lattice paths, concatenations of coordinate steps

$$
\pi^{i}:=\pi^{\varepsilon(i)}, \quad \pi^{\bar{i}}:=\pi^{-\varepsilon(i)}
$$

for $i \in[1, n]$. A subset $\tau=\{\tau(1)<\cdots<\tau(i)\} \subset[1,2 n]$ corresponds to the lattice path $\pi(\tau):=\pi^{\tau(1)} * \cdots * \pi^{\tau(r)}$. We write:

$$
\pi[1, i]:=\pi([1, i])=\pi^{1} * \pi^{2} * \cdots * \pi^{i} .
$$

Any path obtained from $\pi[1, i]$ by repeated application of the lowering operators $f_{1}, \ldots, f_{n}$ is of the form $\pi(\tau)$ for some $\tau$, and we say such paths $\pi(\tau)$ (or subsets $\tau)$ are lattice-standard of shape $\varpi_{i}$. A basis for $V_{\varpi_{i}}^{*}$ is given by $\left\{p_{\tau} \mid \tau\right.$ lattice-standard $\}$. This basis has the triangularity property of Theorem 3 . For more details, see [2].

Similarly, a path $\Pi$ is lattice-standard for $V_{\mathbf{i}, \mathbf{m}}^{*}$ if it can be constructed by the usual formula, with the $\pi^{\lambda_{i}}$ replaced by dominant lattice-paths:

$$
\Pi=f_{i_{1}}^{l_{1}}\left(\pi\left[1, i_{1}\right]^{* m_{1}} * f_{i_{2}}^{l_{2}}\left(\pi\left[1, i_{2}\right]^{* m_{2}} * \cdots f_{i_{r}}^{l_{r}}\left(\pi\left[1, i_{r}\right]^{* m_{r}}\right) \cdots\right)\right),
$$

where $\pi[1, i]^{* m}:=\pi[1, i] * \cdots * \pi[1, i]$ ( $m$ factors). Every such path is a concatenation of 
lattice-standard tableaux for fundamental weights

$$
\Pi=\pi\left(\tau^{(11)}\right) * \pi\left(\tau^{(12)}\right) * \cdots * \pi\left(\tau^{\left(1 m_{1}\right)}\right) * \pi\left(\tau^{(21)}\right) * \cdots * \pi\left(\tau^{\left(r m_{r}\right)}\right),
$$

where $\tau^{(j m)} \subset[1,2 n], \# \tau^{(j m)}=i_{j}$ for $1 \leqslant j \leqslant r, 1 \leqslant m \leqslant m_{j}$. A basis of $V_{\mathbf{i}, \mathbf{m}}^{*}$ is given by

$$
\left\{\rho\left(p_{\tau^{(11)}} \otimes \cdots \otimes p_{\tau^{\left(m m_{r}\right)}}\right) \mid\left(\tau^{(11)}, \ldots, \tau^{\left(r m_{r}\right)}\right) \text { is lattice-standard }\right\},
$$

the restriction of standard tensor-monomials via the map $\rho$. Alternatively, we can realize $V_{\mathbf{i}, \mathbf{m}}^{*}$ inside $\mathbf{k}[G]$, with a basis of monomials in the minors $p_{\tau}$

$$
V_{\mathbf{i}, \mathbf{m}}^{*} \cong \operatorname{Span}_{\mathbf{k}}\left\{p_{\tau^{(11)}} \cdots p_{\tau^{(r m r)}} \mid\left(\tau^{(11)}, \ldots, \tau^{\left(r m_{r}\right)}\right) \text { is lattice-standard }\right\}
$$

EXAMPLE. Let $G=S p_{4}, \mathbf{i}=(2,1,2), \mathbf{m}=(1,1,1)$. For conciseness, we denote the lattice-path $\pi^{a} * \pi^{b} * \pi^{c} * \cdots$ by the list $a b c \cdots$. The lattice-standard tableaux for $V_{\omega_{1}}^{*}$ are $\{1,2, \overline{2}, \overline{1}\}$, meaning $\left\{\pi^{1}, \pi^{2}, \pi^{\overline{2}}, \pi^{\overline{1}}\right\}$; with crystal graph: $1 \stackrel{f_{1}}{\rightarrow} 2 \stackrel{f_{2}}{\rightarrow} \overline{2} \stackrel{f_{1}}{\rightarrow} \overline{1}$. For $V_{\varpi_{2}}^{*}$, the lattice-standard tableaux are: $\{12,1 \overline{2}, 2 \overline{2}, 2 \overline{1}, \overline{2} \overline{1}\}$, meaning $\left\{\pi^{1} * \pi^{2}, \pi^{1} * \pi^{2}, \ldots\right\} ;$ with crystal graph: $12 \stackrel{f_{2}}{\rightarrow} 1 \overline{2} \stackrel{f_{1}}{\rightarrow} 2 \overline{2} \stackrel{f_{1}}{\rightarrow} 2 \overline{1} \stackrel{f_{2}}{\rightarrow} \overline{2} \overline{1}$.

For $V_{\mathbf{i}, \mathbf{m}}^{*}$, we can construct the 17 lattice-standard tableaux of the form $\Pi=f_{2}^{\bullet}\left(12 * f_{1}^{\bullet}\left(1 * f_{2}^{\bullet} 12\right)\right)$ in steps, starting from the right end of the expression for $\Pi$ :

$$
\begin{aligned}
& \{12\} \stackrel{f_{2}^{\bullet}}{\rightarrow}\{12,1 \overline{2}\} \stackrel{1 *}{\rightarrow}\{121,11 \overline{2}\} \stackrel{f_{i}^{\bullet}}{\rightarrow}\{112,11 \overline{2}, 212,21 \overline{2}, 22 \overline{2}, 22 \overline{1}\} \\
& \stackrel{12 *}{\rightarrow}\{12112,1211 \overline{2}, 12212,1221 \overline{2}, 1222 \overline{2}, 1222 \overline{1}\} \\
& \stackrel{f_{2}^{\bullet}}{\rightarrow}\{12112,1211 \overline{2}, 12212,1221 \overline{2}, 1222 \overline{2}, 1222 \overline{1}, 1 \overline{2} 112,1211 \overline{2}, 1 \overline{2} 212, \\
& 1 \overline{2} \overline{2} 12,1 \overline{2} \overline{2} 1 \overline{2}, 1 \overline{2} 21 \overline{2}, 1 \overline{2} 22 \overline{2}, 1 \overline{2} \overline{2} 2 \overline{2}, 1 \overline{2} 22 \overline{1}, 1 \overline{2} \overline{2} 2 \overline{1}, 1 \overline{2} \overline{2} \overline{2} \overline{1}\} \text {. }
\end{aligned}
$$

A list like $1 \overline{2} \overline{2} 2 \overline{1}$ represents the lattice-path $\Pi=\pi^{1} * \pi^{\overline{2}} * \pi^{\overline{2}} * \pi^{2} * \pi^{\overline{1}}$, which can be divided into lattice-standard paths for fundamental weights as: $\Pi=\pi(1 \overline{2}) * \pi(\overline{2}) * \pi(2 \overline{1})$. To illustrate the action of the lowering operator $f_{1}$, we write under each tableau the value of $\left\langle\pi^{j}, \alpha_{1}^{\vee}\right\rangle=-1,0$, or +1 for each coordinate step $\pi^{j}$, and we emphasize the step which is flipped by $f_{1}$ (that is, the step where the path rises for the last time from the minimum value of $\left.\left\langle\pi(t), \alpha_{1}^{\vee}\right\rangle\right)$ :

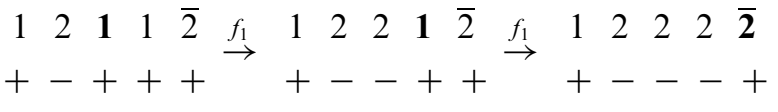

$$
\begin{aligned}
& \begin{array}{llllllll}
f_{1} & \begin{array}{llllll}
1 & 2 & 2 & 2 & 1 & f_{1} \\
& + & - & - & - & -
\end{array} & &
\end{array} \text { undefined }
\end{aligned}
$$

The 17 standard tableaux index a basis for $V_{\mathbf{i}, \mathbf{m}}^{*}$, which we can realize inside $\mathbf{k}[G]$, the coordinate ring of $G \subset G L_{4}$. That is, $\mathbf{k}[G]$ is the polynomial ring $\mathbf{k}\left[x_{i j}\right]_{i, j \in[1,4]}$, where $X=\left(x_{i j}\right)$ a generic matrix, modulo the ideal generated by the supra-diagonal 
coordinates of $X E X^{t}-E$ : that is, modulo the polynomials

$$
\begin{aligned}
& -x_{41} x_{12}-x_{31} x_{22}+x_{21} x_{32}+x_{11} x_{42}, \quad-x_{41} x_{13}-x_{31} x_{23}+x_{21} x_{33}+x_{11} x_{43}, \\
& -x_{41} x_{14}-x_{31} x_{24}+x_{21} x_{34}+x_{11} x_{44}-1, \\
& -x_{42} x_{13}-x_{32} x_{23}+x_{22} x_{33}+x_{12} x_{43}-1, \\
& -x_{42} x_{14}-x_{32} x_{24}+x_{22} x_{34}+x_{12} x_{44}, \quad-x_{43} x_{14}-x_{33} x_{24}+x_{23} x_{34}+x_{13} x_{44} .
\end{aligned}
$$

(This ideal has a small square-free Grobner basis in the degree-lex order, so it is reduced.) For example, the tableau $1 \overline{2} \overline{2} 2 \overline{1}$ corresponds to the following polynomial in $\mathbf{k}[G]$ :

$$
p_{1 \overline{2} \overline{2} 2 \overline{1}}=p_{1 \overline{2}} p_{\overline{2}} p_{2 \overline{1}}=\left(x_{11} x_{32}-x_{31} x_{12}\right) \cdot x_{31} \cdot\left(x_{21} x_{42}-x_{41} x_{22}\right) .
$$

(Recall $\overline{2}=3, \overline{1}=4$.)

\section{Combinatorics of Tableaux}

In this section, we regard the word $\mathbf{i}=\left(i_{1}, \ldots, i_{r}\right)$ as given, and for $J \subset[1, r]$, we abbreviate $w(\mathbf{i}(J))$ as $w(J)$. For example, $s w(J)$ means $s \cdot w(\mathbf{i}(J))$.

\subsection{THE MAIN LEMMA}

LEMMA 7. Consider a long list of Weyl group cosets

$$
\left(\tau_{11}, \ldots, \tau_{r p_{r}}\right)=\left(\tau_{1}, \ldots, \tau_{N}\right)
$$

possessing a lifting

$$
\left(J_{11} \supset \cdots \supset J_{r p_{r}}\right)=\left(J_{1} \supset \cdots \supset J_{N}\right),
$$

meaning that each subword $\mathbf{i}\left(J_{j p}^{(j)}\right)$ is reduced and $\tau_{j p} \equiv w\left(J_{j p}^{(j)}\right) \bmod W_{\lambda_{j}}$. Suppose that for some consecutive elements $\tau_{j p}=\tau_{K-1}, \tau_{j^{\prime} p^{\prime}}=\tau_{K}, \tau_{j^{\prime \prime} p^{\prime \prime}}=\tau_{K+1}$, and for some simple reflection $s$ we have either:

(a) $\quad s w\left(J_{K-1}^{(j)}\right)<w\left(J_{K-1}^{(j)}\right)$ and $s \tau_{K} \geqslant \tau_{K} ;$ or

(b) $s w\left(J_{K+1}^{\left(j^{\prime \prime}\right)}\right)>w\left(J_{K+1}^{\left(j^{\prime \prime}\right)}\right)$ and $s \tau_{K} \leqslant \tau_{K}$.

Then the long list of cosets

$$
\left(\tau_{1}, \ldots, \tau_{K-1}, s \tau_{K}, \tau_{K+1}, \ldots, \tau_{N}\right)
$$

also possesses a lifting $\left(\tilde{J}_{1} \supset \cdots \supset \tilde{J}_{N}\right)$, with (respectively):

(a') $s w\left(\tilde{\boldsymbol{J}}_{K}^{\left(j^{\prime}\right)}\right)<w\left(\tilde{\boldsymbol{J}}_{K}^{\left(j^{\prime}\right)}\right) ; \quad$ or

(b') $\operatorname{sw}\left(\tilde{\boldsymbol{J}}_{K}^{\left(j^{\prime}\right)}\right)>w\left(\tilde{\boldsymbol{J}}_{K}^{\left(j^{\prime}\right)}\right)$.

Proof (cf. [19, Pf of Thm 10.1]). We will prove the lemma under assumption (a). For (b), replace $<$ by $>$ and $K-1$ by $K+1$. First suppose $s w\left(J_{K}^{\left(i^{\prime}\right)}\right)<w\left(J_{K}^{\left(j^{\prime}\right)}\right)$. Then we must have $s \tau_{K}=\tau_{K}$, and we may take $\tilde{J}_{k}=J_{k}$ for all $k$. 
On the other hand, suppose $s w\left(J_{K}^{\left(j^{\prime}\right)}\right)>w\left(J_{K}^{\left(j^{\prime}\right)}\right)$. We have

$$
\operatorname{sw}\left(J_{K-1}^{(j)}\right)<w\left(J_{K-1}^{(j)}\right) \geqslant w\left(J_{K}^{(j)}\right),
$$

and by the Zigzag Lemma [5, Proposition 5.9], $s w\left(J_{K}^{(j)}\right) \leqslant w\left(J_{K-1}^{(j)}\right)$. Thus by the subword definition of Bruhat order, there is a reduced $J_{K}^{\prime} \subset J_{K-1}^{(j)}$ with $w\left(J_{K}^{\prime}\right)=s w\left(J_{K}^{(j)}\right)$. Furthermore for all $L>K$ there are reduced subwords $J_{L}^{\prime} \subset J_{K-1}^{(j)}$ with $w\left(J_{L}^{\prime}\right)=w\left(J_{L}^{(j)}\right)$, and we may take the sets $J_{L}^{\prime}$ to be decreasing as $L$ increases. Now define $\widetilde{J}_{L}=J_{L}$ for $L<K$ and $\widetilde{J}_{L}=\left(J_{L} \backslash[1, j]\right) \cup J_{L}^{\prime}$ for $L \geqslant K$.

Now

$$
w\left(\widetilde{J}_{K}^{\left(j^{\prime}\right)}\right)=w\left(J_{K}^{\prime}\right) w\left(J_{K} \cap\left[j+1, j^{\prime}\right]\right)=s \cdot w\left(J_{K}^{(j)}\right) \cdot w\left(J_{K} \cap\left[j+1, j^{\prime}\right]\right),
$$

and by our supposition the latter product is reduced (length of product $=$ sum of lengths). Hence $\widetilde{J}_{K}^{\left(i^{\prime}\right)}$ is a reduced word, and $w\left(\widetilde{J}_{K}^{\left(i^{\prime}\right)}\right)=s w\left(J_{K}^{\left(j^{\prime}\right)}\right) \equiv s \tau_{K}$. Similarly the appropriate initial segment of $\widetilde{J}_{L}$ for any other $L$ is a reduced lifting of $\tau_{L}$. Therefore $\left(\widetilde{J}_{1} \supset \cdots \supset \widetilde{J}_{N}\right)$ is a lifting of $\left(\tau_{1}, \ldots, s \tau_{K}, \ldots, \tau_{N}\right)$ as required. Property $\left(a^{\prime}\right)$ follows from the above along with our supposition.

\subsection{ROOT OPERATORS}

LEMMA 8. Let $i \in[1, n]$.

(a) If $\Pi$ is a liftable tableau, and $e_{i}(\Pi)$ exists, then $e_{i}(\Pi)$ is liftable.

(b) If $\Pi$ is a liftable tableau, and $e_{i}(\Pi), f_{i}(\Pi)$ both exist, then $f_{i}(\Pi)$ is liftable.

(c) If $\Pi$ is a liftable tableau with respect to $\mathbf{i}$, and $f_{i_{1}}(\Pi)$ exists, where $i_{1}$ is the first letter of i, then $f_{i_{1}}(\Pi)$ is liftable.

Proof. It is clear by [18] that $e_{i}(\Pi), f_{i}(\Pi)$ are always tableaux if they are defined, so in each case we need only show liftability.

(a) Suppose

$$
\bar{\Pi}=\left(\tau_{11}, \ldots, \tau_{r p_{r}}\right)=\left(\tau_{1}, \ldots, \tau_{N}\right)
$$

with lifting

$$
\left(J_{11} \supset \cdots \supset J_{r p_{r}}\right)=\left(J_{1} \supset \cdots \supset J_{N}\right) .
$$

It is easily seen (cf. [17, Proposition 4.2]) that

$$
\overline{e_{i}(\Pi)}=\left\{\begin{array}{l}
\left(\tau_{1}, \ldots, \tau_{K-1}, s \tau_{K}, \ldots, s \tau_{L}, \tau_{L+1}, \ldots \tau_{N}\right) \quad \text { or } \\
\left(\tau_{1}, \ldots, \tau_{K-1}, \tau_{K}, s \tau_{K}, \ldots, s \tau_{L}, \tau_{L+1}, \ldots \tau_{N}\right)
\end{array}\right.
$$

for some indices $1 \leqslant K \leqslant L \leqslant N$ with

$$
s \tau_{K} \leqslant \tau_{K}, \ldots, s \tau_{L} \leqslant \tau_{L} \quad \text { and } \quad s \tau_{L+1}>\tau_{L+1} .
$$

We must show that this list of cosets has a lifting. 
First, suppose $L \neq N$, so that $\tau_{L+1}=\tau_{j^{\prime \prime} p^{\prime \prime}}$ exists, and let $\tau_{L}=\tau_{j^{\prime} p^{\prime}}$. Then $s w\left(J_{L+1}^{j^{\prime \prime}}\right)>w\left(J_{L+1}^{j^{\prime \prime}}\right)$ and $s \tau_{L} \leqslant \tau_{L}$, so we can apply Lemma $7(\mathrm{~b})$ to the lifting $\left(J_{1} \supset \cdots\right)$ at positions $L, L+1$. Using condition $\left(\mathrm{b}^{\prime}\right)$ we can repeat this at positions $L-1, L$, and so on leftward, thus producing a lifting of $e_{i}(\Pi)$.

On the other hand, suppose $L=N$. If $s w\left(J_{L}^{\left(i^{\prime}\right)}\right)>w\left(J_{L}^{\left(j^{\prime}\right)}\right)$, then $\tau_{N}=s \tau_{N}$, and we may again repeatedly apply the Lemma starting at positions $N-1, N$. If $s w\left(J_{N}^{\left(j^{\prime}\right)}\right)<w\left(J_{N}^{\left(j^{\prime}\right)}\right)$, we cannot directly apply the Lemma, but instead take $\tilde{J}_{N} \subset J_{N}$ so that $w\left(\tilde{\boldsymbol{J}}_{N}^{\left(j^{\prime}\right)}\right)=\operatorname{sw}\left(J_{N}^{\left(j^{\prime}\right)}\right)$. Then $\left(J_{1} \supset \cdots \supset J_{N-1} \supset \tilde{J}_{N}\right)$ is a lifting of $\left(\tau_{1}, \ldots, \tau_{N-1}, s \tau_{N}\right)$, to which we can apply the Lemma starting at positions $N-1$, $N$. In each case, we produce a lifting of $e_{i}(\Pi)$.

(b) We have

$$
\overline{f_{i}(\Pi)}=\left\{\begin{array}{l}
\left(\tau_{1}, \ldots, \tau_{K-1}, s \tau_{K}, \ldots, s \tau_{L}, \tau_{L+1}, \ldots \tau_{N}\right) \quad \text { or } \\
\left(\tau_{1}, \ldots, \tau_{K-1}, s \tau_{K}, \ldots, s \tau_{L}, \tau_{L}, \tau_{L+1}, \ldots \tau_{N}\right)
\end{array}\right.
$$

for $1 \leqslant K \leqslant L \leqslant N$ with

$$
s \tau_{K-1}<\tau_{K-1} \text { and } s \tau_{K} \geqslant \tau_{K}, \ldots, s \tau_{L} \geqslant \tau_{L} .
$$

Since $e_{i}(\Pi)$ also exists, we must have $K>1$, so that we may repeatedly apply Lemma 7(a) analogously to the previous argument, starting at positions $K-1, K$ and proceeding rightward to produce a lifting of $f_{i}(\Pi)$.

(c) Let $J_{K}=J_{j^{\prime} p^{\prime}}$. As before, if $K>1$ or $s w\left(J_{1}^{\left(j^{\prime}\right)}\right)<w\left(J_{1}^{\left(j^{\prime}\right)}\right)$, we use Lemma 7(a) immediately. Otherwise if $K=1$ and $s w\left(J_{1}^{\left(j^{\prime}\right)}\right)>w\left(J_{1}^{\left(i^{\prime}\right)}\right)$, take $\tilde{J}_{1}=J_{1} \cup\{1\}$ (reduced): this gives a lifting $\left(\tilde{J}_{1} \supset J_{2} \supset \cdots \supset J_{N}\right)$ of $\left(s \tau_{1}, \tau_{2}, \ldots, \tau_{N}\right)$, to which we apply Lemma 7(a).

Proof of Theorem 1. We use induction on $r$, the number of letters of the word $\mathbf{i}=\left(i_{1}, \ldots, i_{r}\right)$. For $r=0$ and $\mathbf{i}$ the empty word, the only constructable or liftable tableau is the trivial path $\pi^{0}$. Now assume the Theorem for the word $\left(i_{2}, \ldots, i_{r}\right)$.

Constructable $\Rightarrow$ liftable. Suppose $\Pi=f_{i_{1}}^{l_{1}}\left(\pi^{\lambda_{1}} * f_{i_{2}}^{l_{2}}\left(\pi^{\lambda_{2}} \ldots\right)\right)$ is constructable. By induction $\Pi^{\prime}=f_{i_{2}}^{l_{2}}\left(\pi^{\lambda_{2}} \ldots\right)$ is liftable. Then $\pi^{\lambda_{1}} * \Pi^{\prime}$ is clearly also liftable, and so is $\Pi=f_{i_{1}}^{l_{1}}\left(\pi^{\lambda_{1}} * \Pi^{\prime}\right)$ by Lemma $8(\mathrm{c})$.

Liftable $\Rightarrow$ constructable. Suppose $\Pi=\left(\pi_{1}, \ldots, \pi_{r}\right)$ has lifting $\left(J_{11} \supset \cdots\right)$. Since $w\left(J_{1 p}^{(1)}\right)=s_{i_{1}}$ or id, the cosets $\bmod W_{\lambda_{1}}$ in the LS-chain $\pi_{1}$ must be $\overline{\pi_{1}}=\left(s_{i_{1}}, \ldots, s_{i_{1}}\right.$, id,$\ldots$, id $)$. Thus we may write $e_{i_{1}}^{\text {top }}(\Pi)=e_{i_{1}}^{l_{1}}(\Pi)=\left(\pi^{\lambda_{1}}, \pi_{2}^{\prime}, \ldots, \pi_{r}^{\prime}\right)$ for some $l_{1}$, since if the initial segment were not $\pi^{\lambda_{1}}$, we could apply $e_{i_{1}}$ once more (Section 1.2). But $\Pi^{\prime}=\left(\pi_{2}^{\prime}, \ldots, \pi_{r}^{\prime}\right)$ is liftable by Lemma $8(\mathrm{a})$, and is therefore constructable by induction. Hence $\Pi=f_{i_{1}}^{l_{1}}\left(\pi^{\lambda_{1}} * \Pi^{\prime}\right)$ is also constructable.

\subsection{DEMAZURE OPERATORS}

We show that the number of liftable tableaux is given by the Demazure character formula. This is the combinatorial version of Theorem 5. For a set $\mathcal{T}$ of tableaux, define the formal character (or multi-variate generating function) 
$\operatorname{char}(\mathcal{T}):=\sum_{\Pi \in \mathcal{T}} \mathrm{e}^{\mathrm{wt}(\Pi)}$. For $i \in[1, n]$, a tableau $\Pi_{0}$ with $e_{i}\left(\Pi_{0}\right)$ undefined is called an $i$-head. An $i$-string is the set $S$ of all tableaux generated under $f_{i}$ by some $i$-head $\Pi_{0}$ : $S=\left\{\Pi_{0}, f_{i}\left(\Pi_{0}\right), f_{i}^{2}\left(\Pi_{0}\right), \ldots, f_{i}^{l}\left(\Pi_{0}\right)\right\}$, where $l$ is maximal with $f_{i}^{l}\left(\Pi_{0}\right)$ defined. In fact $l=\left\langle\operatorname{wt}\left(\Pi_{0}\right), \alpha_{i}^{\vee}\right\rangle \geqslant 0$. (See [18].) Thus any $i$-string $S$ with head $\Pi_{0}$ has character

$$
\operatorname{char}(S)=\mathrm{e}^{\mathrm{wt}\left(\Pi_{0}\right)}+\mathrm{e}^{\mathrm{wt}\left(\Pi_{0}\right)-\alpha_{i}}+\cdots+\mathrm{e}^{s_{i} \mathrm{wt}\left(\Pi_{0}\right)}
$$

Suppose a set of tableau $\mathcal{T}$ is a disjoint union of $i$-heads and $i$-strings. Let

$$
f_{i}^{\bullet \mathcal{T}}:=\left\{f_{i}^{l}(\Pi) \mid \Pi \in \mathcal{T}, l \geqslant 0\right\} .
$$

Then it is clear that $\operatorname{char}\left(f_{i}^{\bullet} \mathcal{T}\right)=\Lambda_{i}(\operatorname{char} \mathcal{T})$.

LEMMA 9. Let $\mathcal{T}$ be the set of standard tableau of shape $\left(\lambda_{2}, \ldots, \lambda_{r}\right)$. Then the set of concatenations $\pi^{\lambda_{1}} * \mathcal{T}$ is a disjoint union of $i_{1}$-heads and $i_{1}$-strings.

Proof. Let $\pi_{1}=\pi^{\lambda_{1}}, e=e_{i_{1}}, f=f_{i_{1}}$. First we show: if $\pi_{1} * \Pi \in \pi * \mathcal{T}$ with $e\left(\pi_{1} * \Pi\right)$ defined, then $e\left(\pi_{1} * \Pi\right), f\left(\pi_{1} * \Pi\right) \in \pi_{1} * \mathcal{T}$. By [18, $\left.\S 2.6\right]$ we have $e\left(\pi_{1} * \Pi\right)=\pi_{1} *(e \Pi)$ or $\left(e \pi_{1}\right) * \Pi$; but $e \pi_{1}$ is undefined, so the first alternative must hold. Also $e \Pi \in \mathcal{T}$ by Lemma 8 (a), so $e\left(\pi_{1} * \Pi\right) \in \pi_{1} * \mathcal{T}$.

Next we show that if $\pi_{1} * \Pi \in \pi_{1} * \mathcal{T}$ with $e\left(\pi_{1} * \Pi\right)$ and $f\left(\pi_{1} * \Pi\right)$ are both defined, then $f\left(\pi_{1} * \Pi\right) \in \pi_{1} * \mathcal{T}$. First, recall that the operator $e$ reflects a part of $\pi_{1} * \Pi$ before the first minimum point of the function $t \mapsto\left\langle\left(\pi_{1} * \Pi\right)(t), \alpha^{\vee}\right\rangle$, and the operator $f$ reflects a part of $\pi_{1} * \Pi$ after the last minimum point of the function. Thus $f$ acts on the path at a later point than $e$ acts.

Now consider $f\left(\pi_{1} * \Pi\right)=\left(f \pi_{1}\right) * \Pi$ or $\pi_{1} *(f \Pi)$. We know that $e\left(\pi_{1} * \Pi\right)=\pi_{1} *(e \Pi)$, so that $e$ acts after the first segment of $\pi_{1} * \Pi$; and $f$ acts later than $e$. Thus we must have the second alternative: $f\left(\pi_{1} * \Pi\right)=\pi_{1} *(f \Pi)$. But $f \Pi \in \mathcal{T}$ by Lemma 8 (b), so $f\left(\pi_{1} * \Pi\right) \in \pi_{1} * \mathcal{T}$.

COROLLARY 10. The formal character of the set of standard tableaux of shape $\left(\lambda_{1}, \ldots, \lambda_{r}\right)$ is

$$
\Lambda_{i_{1}}\left(\mathrm{e}^{\lambda_{1}} \Lambda_{i_{2}}\left(\mathrm{e}^{\lambda_{2}} \cdots \Lambda_{i_{r}}\left(\mathrm{e}^{\lambda_{r}}\right) \cdots\right)\right)
$$

Proof. Let $\mathcal{T}$ be the set of standard tableaux for the $\left(i_{2}, \ldots, i_{r}\right)$. The set of standard tableaux for $\left(i_{1}, \ldots, i_{r}\right)$ is $\mathcal{T}^{\prime}=f_{i_{1}}^{\bullet}\left(\pi^{\lambda_{1}} * \mathcal{T}\right)$, so that char $\mathcal{T}^{\prime}=\Lambda_{i_{1}}\left(\mathrm{e}^{\lambda_{1}} \operatorname{char} \mathcal{T}\right)$. The result now follows by induction.

\subsection{COMBINATORIAL EXCELLENT FILTRATION}

The ordinary Demazure modules $V_{\lambda}(w)$ and their duals play a central role in the theory of $B$-modules. For example, consider the twisted dual Demazure module $\mathbf{k}_{\mu}^{*} \otimes V_{v}^{*}(y)$ for dominant weights $\mu, v \in X^{+}$. It was conjectured by A. Joseph and proved by O. Mathieu [25] (in the general case, and by P. Polo in some special cases) 
that this module has an excellent filtration, namely a filtration by $B$-modules whose quotients are isomorphic to $V_{\lambda}^{*}(w)$ for various $\lambda \in X^{+}, w \in W$. This implies that $\mathbf{k}_{\mu}^{*} \otimes V_{v}^{*}(y)$ inherits the favorable homological properties of the $V_{\lambda}^{*}(w)$. More generally, Mathieu proved that twists of our generalized Demazure modules $\mathbf{k}_{\mu}^{*} \otimes V_{\mathbf{i}, \mathbf{m}}^{*}$ have excellent filtration. For a survey, see [27].

In this section, we prove Theorem 2, which is a combinatorial analogue of Mathieu's result. We first formulate and prove a more precise result for the special case of a twisted ordinary Demazure module $\mathbf{k}_{\mu}^{*} \otimes V_{v}^{*}(y)$. (Note that we do not distinguish between tableaux for this module and for its dual.)

Let $C(\lambda, w)$ denote the crystal graph on the set $\left\{f_{i_{1}}^{\bullet} \cdots f_{i_{r}}^{\bullet} \pi^{\lambda}\right\}$ of standard tableaux for $V_{\lambda}(w)$, where $w=s_{i_{1}} \cdots s_{i_{r}}$ is any reduced decomposition. This set of tableaux is known to be independent of the choice of reduced decomposition. In fact, it is precisely the set of LS-paths $\pi=\left(\tau_{1}>\cdots ; 0<a_{1} \cdots\right)$ such that $w \geqslant \tau_{1} W_{\lambda}$. See $[7,17]$.

Further, let $C(\mu, v, y)$ be the crystal graph on the set $\left\{\pi^{\mu} * \pi\right\}$, where $\pi$ runs over standard tableaux for $V_{v}(y)$. Recall that each edge in a crystal graph is assigned a color $i=1, \ldots, n$. We will show that for dominant $\mu, v$, the $C(\mu, v, y)$ is isomorphic as an edge-colored graph to a disjoint union of various $C(\lambda, w)$. There is one such component $C(\lambda, w)$ for each path $\pi^{\mu} * \pi$ which is dominant, i.e., which stays completely within the dominant Weyl chamber: that is, $\left\langle\mu+\pi(t), \alpha_{i}^{\vee}\right\rangle \geqslant 0$ for all $t \in[0,1]$ and all $i$. The $\lambda$ corresponding to a dominant path is its weight: $\lambda=\operatorname{wt}\left(\pi^{\mu} * \pi\right)=\mu+\operatorname{wt}(\pi)$. To compute the corresponding $w$ requires some definitions.

The second part of the following lemma is due to Deodhar [14, Lemma 11.1].

LEMMA 11 (i) For $u, w \in W$, the set $\left\{u^{\prime} w \mid u^{\prime} \leqslant u\right\}$ has unique maximal and minimal elements.

(ii) Let $W^{\prime} \subset W$ be the parabolic subgroup generated by some subset of the simple roots. For $z \leqslant y \in W$, the set $\left\{u^{\prime} z \mid u^{\prime} \in W^{\prime}, u^{\prime} z \leqslant y\right\}$ has a unique maximal element.

Proof. For a set $A \subset W$, we write $w=\max A$ if $w \in A$ and $w \geqslant y$ for all $y \in A$ (that is, $w$ is the unique Bruhat-maximal element of $A$ ). Similarly for $\min A$. We will repeatedly use the Zigzag Lemma [5, § 5.11]:

If $x \leqslant y$, then $s x \leqslant \max (y, s y)$ and $\min (x, s x) \leqslant s y$,

where $s$ is any simple reflection, and similarly for $x s$ and $y s$.

(i) Induction on $\ell(u)$. The case $\ell(u)=0$ is trivial. Take $s u>u$, and assume $u^{\prime} w \leqslant u_{1} w$ for all $u^{\prime} \leqslant u$. We claim $u^{\prime \prime} w \leqslant \max \left(u_{1} w, s u_{1} w\right)$ for all $u^{\prime \prime} \leqslant s u$, so that $\max \left(u_{1} w, s u_{1} w\right)$ is the unique maximum of $\left\{u^{\prime \prime} w \mid u^{\prime \prime} \leqslant s u\right\}$.

From the subword definition of Bruhat order, any $u^{\prime \prime} \leqslant s u$ has either: $u^{\prime \prime} \leqslant u$, so that $u^{\prime \prime} w \leqslant u_{1} w ; \quad$ or $\quad s u^{\prime \prime} \leqslant u$, so that $\quad s u^{\prime \prime} w \leqslant u_{1} w, \quad$ and $\quad u^{\prime \prime} w=s\left(s u^{\prime \prime} w\right) \leqslant$ $\max \left(u_{1} w, s u_{1} w\right)$ by the Zigzag Lemma. This proves the claim.

The proof that $\left\{u^{\prime} w \mid u^{\prime} \leqslant u\right\}$ has a unique minimum is almost the same: Again take $s u>u$. If $u_{0} w \leqslant u^{\prime} w$ for all $u^{\prime} \leqslant u$, then $\min \left(u_{0} w, s u_{0} w\right) \leqslant u^{\prime \prime} w$ for $u^{\prime \prime} \leqslant s u$. 
(ii) We follow Deodhar [14, Lemma 11.1], correcting several misprints. We denote $\left(W^{\prime} z\right)_{\leqslant y}:=\left\{u^{\prime} z \mid u^{\prime} \in W^{\prime}, u^{\prime} z \leqslant y\right\}$. Also let $W^{\text {min }} \subset W$ be the set of minimal coset representatives of $W^{\prime} \backslash W$, and write $y=w y_{0}$ with $w \in W^{\prime}$ and $y_{0} \in W^{\min }$. Suppose, without loss of generality, that $z=z_{0} \in W^{\min }$ (since $\left(W^{\prime} u z\right)_{\leqslant y}=\left(W^{\prime} z\right)_{\leqslant y}$ for any $\left.u \in W^{\prime}\right)$. Now we proceed by induction on $\ell\left(y_{0}\right)$. For $\ell\left(y_{0}\right)=0$, we have $y_{0}=z_{0}=e$, and $\max \left(W^{\prime} z\right)_{\leqslant y_{0} w}=w$. Next suppose $\ell\left(y_{0}\right)>0$, and choose a simple root $s$ with $y_{0}<y_{0} s$. Note that $y_{0} s \in W^{\mathrm{min}}$, since otherwise there is a simple root $s^{\prime} \in W^{\prime}$ with $\ell\left(s^{\prime} y_{0} s\right)=\ell\left(y_{0}\right)-2=\ell\left(s^{\prime} y_{0}\right)-3$. Note also that $w y_{0} s<w y_{0}$, since: $\ell\left(w y_{0} s\right)=\ell(w)+\ell\left(y_{0} s\right)=\ell(w)+\ell\left(y_{0}\right)-1=\ell\left(w y_{0}\right)-1$.

Case (a): $z_{0} s<z_{0}$. The facts noted above for $y_{0}$ also hold for $z_{0}$. We have $z_{0} s=\min \left(z_{0} s, z_{0}\right) \leqslant y_{0} s$, and by induction we may let $w^{\prime} z_{0} s=\max \left(W^{\prime} z_{0} s\right)_{\leqslant w y_{0}}$. Then we claim $w^{\prime} z_{0}=\max \left(W^{\prime} z_{0}\right)_{\leqslant w y_{0}}$. First, $\quad w^{\prime} z_{0} s \leqslant w y_{0} s, \quad$ so $w^{\prime} z_{0} \leqslant \max \left(w y_{0} s, w y_{0}\right)=w y_{0}$. Now suppose $u z_{0} \leqslant w y_{0}$ for $u \in W^{\prime}$. Then $u z_{0} s=\min \left(u z_{0} s, u z_{0}\right) \leqslant w y_{0} s$ and by definition of $w^{\prime}$, we have $u z_{0} s \leqslant w^{\prime} z_{0} s$. Thus $u \leqslant w^{\prime}$ and $u z_{0} \leqslant w^{\prime} z_{0}$.

Case (b): $z_{0} s>z_{0}$ and $z_{0} s \in W^{\text {min }}$. We have $z_{0}=\min \left(z_{0}, z_{0} s\right) \leqslant y_{0} s$, so by

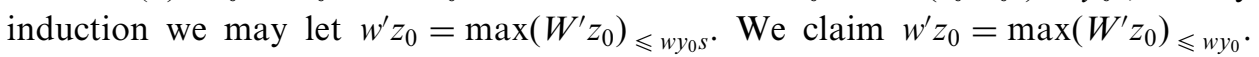
First, $w^{\prime} z_{0} \leqslant w y_{0}$ just as before. Also note that, as before, for any $u \in W^{\prime}$ we have $u z_{0} s>u z_{0}$. Now suppose $u z_{0} \leqslant w y_{0}$ for $u \in W^{\prime}$. Then $u z_{0}=\min \left(u z_{0} s, u z_{0}\right) \leqslant w y_{0} s$ and by definition of $w^{\prime}$, we have $u z_{0} \leqslant w^{\prime} z_{0}$.

Case (c): $z_{0} s>z_{0}$ and $z_{0} s \notin W^{\text {min }}$. First note: there exists a simple root $s^{\prime} \in W^{\prime}$ with $s^{\prime} z_{0} s<z_{0} s, \quad$ so $\quad \ell\left(s^{\prime} z_{0} s\right)=\ell\left(z_{0}\right) ; \quad$ but $\quad s z_{0} \geqslant z_{0}, \quad$ so $\quad s z_{0} s^{\prime} \geqslant \min \left(z_{0}, z_{0} s^{\prime}\right)=z_{0}$. Hence, $s z_{0} s^{\prime}=z_{0}$. Now, as in case (b), we may let $w^{\prime}=\max \left(W^{\prime} z_{0}\right)_{\leqslant w y_{0}}$. Define $\quad w^{\prime \prime}:=\max \left(w^{\prime}, w^{\prime} s^{\prime}\right)$. Then we claim $w^{\prime \prime}=\max \left(W^{\prime} z_{0}\right)_{\leqslant w y_{0}}$. First, $w^{\prime \prime} z_{0}=\max \left(w^{\prime} z_{0}, w^{\prime} z_{0} s\right) \leqslant \max \left(w y_{0}, w y_{0} s\right)=w y_{0}$. Now suppose $u z_{0} \leqslant w y_{0}$ for $u \in W^{\prime}$. If $u s^{\prime}<u$, then $u z_{0}>u s^{\prime} z_{0}=u z_{0} s$ and $u s^{\prime} z_{0}=\min \left(u z_{0}, u z_{0} s\right) \leqslant w y_{0} s$, so by the definition of $w^{\prime}$, we have $u s^{\prime} z_{0} \leqslant w^{\prime} z_{0}$. Hence $u \leqslant w^{\prime \prime}$, and $u z_{0} \leqslant w^{\prime \prime} z_{0}$. On the other hand, if $u s^{\prime}>u$, then $u z_{0}=\min \left(u z_{0}, u z_{0} s\right) \leqslant w y_{0} s$, so by the definition of $w^{\prime}$, we have again $u z_{0} \leqslant w^{\prime} z_{0} \leqslant w^{\prime \prime} z_{0}$.

Now, given $\mu, v, y$ as above, and a path $\pi:[0,1] \rightarrow X_{\mathbb{R}}$, let $W(t):=\operatorname{Stab}_{W}(\mu+\pi(t))$ denote the stabilizer of the point $\mu+\pi(t) \in X_{\mathbb{R}}$. This is the parabolic subgoup of $W$ generated by the simple reflections $s_{i}$ such that $\mu+\pi(t)$ lies on the corresponding wall of the dominant Weyl chamber. Let $[0,1]=I_{1} \sqcup \cdots \sqcup I_{q}$ be the decomposition of $[0,1]$ into the minimal number of disjoint intervals such that $W(t)$ is constant for all $t$ in each interval $I_{j}$. We enumerate the intervals so that for $j<j^{\prime}$, the $t$ in $I_{j}$ are smaller than those in $I_{j^{\prime}}$.

For an LS-path $\pi=\left(\tau_{1}>\cdots ; 0<a_{1}<\cdots\right)$ with $\pi^{\mu} * \pi$ dominant, we let $\lambda=\mu+\mathrm{wt}(\pi)$, and we define $w(\pi)$ (modulo $\left.W_{\lambda}\right)$ inductively as follows. Intuitively, we start with $w=\mathrm{id}$; then we travel along the path $\pi^{\mu} * \pi$ from its endpoint $\lambda$ to $\mu$, and every time we hit a wall, we multiply $w$ by the corresponding reflection if this makes $w$ longer. However, at the end of our trip, if $\mu$ itself is on a wall, we only multiply by the corresponding reflection $s$ if $s$ times the initial direction of 
$\pi$ is smaller than $y$. Formally, we define:

$$
\begin{aligned}
& w_{q}:=\max \left(W\left(I_{q}\right)\right), w_{q-1}:=\max \left(W\left(I_{q-1}\right) \cdot w_{q}\right), \ldots, w_{2}:=\max \left(W\left(I_{2}\right) \cdot w_{3}\right), \\
& w_{1}:=\max \left\{u w_{2} \mid u \leqslant u_{1}\right\} ; \quad w(\pi):=w_{1} .
\end{aligned}
$$

Here $u_{1}:=\max \left\{u \in W\left(I_{1}\right) \mid u \tau_{1} \leqslant y W_{v}\right\}$. We explain why $u_{1}$ is well-defined. The path $\pi^{\mu} * \pi$ has a segment in direction $\tau_{1}(v)$ from $\mu$ into the fundamental chamber. Thus if $s_{i} \in W\left(I_{1}\right)=W_{\mu}$, we have $\left\langle\tau(v), \alpha_{i}^{\vee}\right\rangle \geqslant 0$, and $s_{i} \tau_{1} \geqslant \tau_{1} W_{v}$. That is, we may take $\tau_{1}$ to be minimal in the coset $W\left(I_{1}\right) \tau_{1}$, so that we may use the Lemma to define $u_{1} \tau_{1}=\max \left\{u^{\prime} \tau_{1} \mid u^{\prime} \in W\left(I_{1}\right), u^{\prime} \tau_{1} \leqslant y\right\}$. (Note: In the Kac-Moody setting, in which $W$ may be infinite, we can show that $W\left(I_{j}\right)$ is always finite, and the above definition of $w(\pi)$ is still valid.)

The following result is a refinement of the Littlewood-Richardson rule of [17].

PROPOSITION 12. The crystal graph of $\mathbf{k}_{\mu} \otimes V_{v}(y)$ has as its connected components the crystal graphs of ordinary Demazure modules $V_{\lambda}(w)$. Specifically, it is the disjoint union:

$$
C(\mu, v, y)=\coprod_{\pi} C(\mu+\mathrm{wt}(\pi), w(\pi))
$$

running over all standard tableaux $\pi$ for $V_{v}(y)$ such that $\pi^{\mu} * \pi$ is dominant.

Proof. (a) First the containment $\supset$. Fix a path $\pi=\left(\tau_{1}>\cdots>\tau_{q}\right.$; $0<a_{1}<\cdots<a_{q}=1$ ) with with $y \geqslant \tau_{1} W_{\lambda}$, and $\pi^{\mu} * \pi$ dominant. We must show that for some (and hence for any) reduced word $w(\pi)=s_{i_{1}} \cdots s_{i_{r}}$, the path $f_{i_{1}}^{l_{1}} \cdots f_{i_{r}}^{l_{r}}\left(\pi^{\mu} * \pi\right)$, if defined, is of the form $\pi^{\mu} * \theta$ with $\theta=\left(\sigma_{1}>\cdots ; 0<b_{1}<\cdots\right)$ and $y \geqslant \sigma_{1} W_{\lambda}$. We may choose our reduced word compatible with the partial products $w_{k}$ in the definition of $w(\pi)$. That is, for any $p$, we have $w_{k} \geqslant s_{i_{p}} \cdots s_{i_{r}} \geqslant w_{k+1}$ for some $k$.

Claim: $f_{i_{p}}^{l_{p}} \cdots f_{i_{r}}^{l_{r}}\left(\pi^{\mu} * \pi\right)=\pi^{\mu} * \theta_{p}$ for some $\theta_{p}$ with $\theta_{p}(t)=\pi(t)$ for $t \in I_{1} \sqcup \cdots \sqcup I_{k}$. This follows by descending induction on $p$. Indeed, assume the claim for a given $p$ (with $p>1$ ), and suppose $w_{k}>s_{i_{p}} \cdots s_{i_{r}} \geqslant w_{k+1}$. Then on $t \in I_{1} \sqcup \cdots \sqcup I_{k}$, the function $t \mapsto\left\langle\mu+\theta_{p}(t), \alpha_{i_{p-1}}^{\vee}\right\rangle=\left\langle\mu+\pi(t), \alpha_{i_{p-1}}^{\vee}\right\rangle$ is non-negative, and it attains its minimum value 0 at the right endpoint of $I_{k}$. Hence by definition, the operator $f_{i_{p-1}}^{l_{p-1}}$ does not change the path $\pi^{\mu} * \theta_{p}$ within the interval $t \in I_{1} \sqcup \cdots \sqcup I_{k}$, and the claim holds for $p-1$.

Finally, $y \geqslant \sigma$ follows immediately from the claim and the definition of $u_{1}$.

(b) Now the opposite containment $\subset$. Consider any path $\pi^{\mu} * \theta$ (not necessarily dominant), and consider the unique dominant path $\pi^{\mu} * \pi$ such that $f_{i_{1}}^{l_{1}} \cdots f_{i_{r}}^{l_{r}}\left(\pi^{\mu} * \pi\right)=\pi^{\mu} * \theta$ for some reduced word $s_{i_{1}} \cdots s_{i_{r}}=: w$ and $l_{j}>0$. We must show that $w(\pi) \geqslant w$.

If $\pi^{\mu} * \theta$ is itself dominant, there is nothing to prove. Otherwise, let $I_{k+1}, k \geqslant 1$, be the interval where $\mu+\theta(t)$ first exits the fundamental chamber. We will use decreasing induction on $k$ to show the stronger statement $w_{k} \geqslant s_{i_{1}} \cdots s_{i_{r}}$. 
By the definition of the lowering operators, we have $\mu+\theta(t)=\mu+\pi(t)$ for $t \leqslant$ the first point of exit of $\pi^{\mu} * \theta$ from the fundamental chamber. Thus, for $t \in I_{k}$, $\mu+\theta(t)$ lies on all the walls which are crossed in interval $I_{k+1}$. Thus there exists a product $e_{j_{a}}^{m_{a}} \cdots e_{j_{1}}^{m_{1}}$ of $e_{j}$ with $s_{j} \in W\left(I_{k}\right)$, such that $\pi^{\mu} * \theta^{\prime}:=e_{j_{a}}^{m_{a}} \cdots e_{j_{1}}^{m_{1}}\left(\pi^{\mu} * \theta\right)$ lies inside the dominant chamber for $t \in I_{1} \sqcup \cdots \sqcup I_{k+1}$. By induction, $\pi^{\mu} * \theta^{\prime}=f_{i_{p}}^{l_{p}} \cdots f_{i_{r}}^{l_{r}}\left(\pi^{\mu} * \pi\right)$, where $w_{k+1} \geqslant s_{i_{p}} \cdots s_{i_{r}}$. We thus have $\pi^{\mu} * \theta=$ $f_{j_{1}}^{m_{1}} \cdots f_{j_{a}}^{m_{a}} f_{i_{p}}^{l_{l_{p}}} \cdots f_{i_{r}}^{l_{r}}\left(\pi^{\mu} * \pi\right)$, where $w_{k} \geqslant s_{j_{1}} \cdots s_{j_{a}} s_{i_{p}} \cdots s_{i_{r}}$, provided only that $k>1$. If $k=1$, we may assume that $u_{1} \geqslant s_{j_{1}} \cdots s_{j_{a}}$, and the conclusion again follows.

EXAMPLES. We write $\left(\tau_{1} a_{1} \tau_{2} a_{2} \cdots\right)$ for an LS-chain $\left(\tau_{1}>\cdots ; 0<a_{1}<\cdots\right)$ of $V_{v}$, so a chain $\left(\tau_{1} ; 0<1\right)$ with extremal weight $\tau_{1}(v)$ is written simply $\left(\tau_{1}\right)$.

(i) $G=S L_{3}, \mu=2 \varpi_{2}, v=\varpi_{1}+\varpi_{2}, y=s_{1} s_{2}$. There are four components of $C(\mu, v, y): \pi=\left(s_{1} s_{2}\right), C\left(\varpi_{2}, \mathrm{id}\right) ; \pi=\left(s_{1} s_{2} \frac{1}{2} s_{2}\right), C\left(2 \varpi_{1}, \mathrm{id}\right) ; \pi=\left(s_{1}\right), C\left(\varpi_{1}+2 \varpi_{2}, \mathrm{id}\right)$; $\pi=(\mathrm{id}), \quad C\left(\varpi_{1}+3 \varpi_{2}, s_{2}\right)$. Note that for $\pi=\left(s_{1}\right)$ we have $W\left(I_{1}\right)=\left\langle s_{2}\right\rangle$, but $s_{2} \tau_{1} \$ y$, so $w(\pi)=$ id.

(ii) $G=S L_{3}, \quad \mu=2 \varpi_{1}, \quad v=2 \varpi_{1}+3 \varpi_{2}, \quad y=s_{1} s_{2}$. The path $\pi=\left(s_{1} s_{2} \frac{2}{5} s_{1} \frac{2}{3} e\right)$ corresponds to $C\left(2 \varpi_{1}+\varpi_{2}, s_{1} s_{2}\right)$. Note that $\theta=f_{2} f_{1} f_{2}\left(\pi^{\mu} * \pi\right) \in C(\mu, v, y)$, but this is no contradiction, since $\theta=f_{1} f_{2}^{2}\left(\pi^{\mu} * \pi\right)$.

Proof of Theorem 2. This follows immediately by the definition of constructablestandard tableaux and repeated application of the previous Proposition.

\subsection{RAGHAVAN-SANKARAN OPERATORS}

We define certain raising operators on liftable tableaux, different from the root operators above, which we will need in our proof of Theorem 4. (Cf. [26], [20, $\S 4]$.

Given a tableau $\Pi$ and a simple reflection $s$, define $\widehat{s}(\Pi)$ as follows. Let $\bar{\Pi}=\left(\tau_{1}, \ldots, \tau_{N}\right)$. If for some $1 \leqslant K \leqslant N$ we have

$$
\tau_{1} \geqslant s \tau_{1}, \ldots, \tau_{K} \geqslant s \tau_{K}, \tau_{K+1}<s \tau_{K+1},
$$

take $\widehat{s}(\Pi)$ to have the same rational numbers in its LS-chains as $\Pi$ has, but change the cosets to

$$
\overline{\widehat{s}(\Pi)}=\left(s \tau_{1}, \ldots, s \tau_{K}, \tau_{K+1}, \ldots, \tau_{N}\right) .
$$

(In case $s \tau_{K}=\tau_{K+1}$ and these cosets form part of the same LS-chain, for consistency of notation we must combine these two into a single segment: that is, omit $\tau_{K+1}$ and its corresponding rational number.)

PROPOSITION 13. If $\Pi$ is a liftable tableau, then $\widehat{s}(\Pi)$ is also a liftable tableau.

Proof. First we show that $\widehat{s}(\Pi)$ is a tableau. Suppose the coset $\tau_{K}$ in the definition of $\widehat{s}(\Pi)$ occurs in the $j$ th LS-chain $\pi_{j}$ of $\Pi$ : that is, $\tau_{K}=\tau_{j p}$. Then $\widehat{s}(\Pi)=\left(\widehat{s}\left(\pi_{1}\right), \ldots, \widehat{s}\left(\pi_{j}\right), \pi_{j+1}, \ldots, \pi_{r}\right)$. We thus need to show that if 
$\pi=\left(\tau_{1}>\cdots>\tau_{q} ; 0<a_{1}<\cdots\right)$ is an LS-chain, then $\widehat{s}(\pi)=\left(s \tau_{1}>\cdots>\right.$ $\left.s \tau_{L}>\tau_{L+1}>\cdots>\tau_{q} ; 0<a_{1} \cdots\right)$ is also an LS-chain.

If $L=q$, then $\widehat{s}(\pi)=\mathrm{e}^{\text {top }}(\pi)$, where $e$ is the raising root operator corresponding to s. If $L<q$, let $\pi^{\prime}=\left(\tau_{1}>\cdots>\tau_{L+1} ; 0<a_{1}<\cdots<a_{L}<1\right)$, which is an LS-chain by [17, Lemma 3.1]. Then once again $\widehat{s}\left(\pi^{\prime}\right)=\mathrm{e}^{\text {top }}\left(\pi^{\prime}\right)$, so that $\widehat{s}\left(\pi^{\prime}\right)$ is an LS-chain, and this easily implies that $\widehat{s}(\pi)$ is also an LS-chain. Therefore $\widehat{s}(\Pi)$ is a tableau.

Now to see that $\widehat{s}(\Pi)$ is liftable, we use Lemma 7(b) repeatedly, starting with the positions $L, L+1$ (or $N-1, N$ if $L=N$ ) and proceeding leftward. (Cf. the proof of Lemma 8(a).)

\section{Linear Independence}

\subsection{LS-PATH BASIS}

In order to show the independence of the set $\left\{p_{\Pi}\right\}$ of standard monomials in $V_{\mathbf{i}, \mathbf{m}}^{*}$, we first establish independence for a set $\left\{v_{\Pi}\right\}$ in $V_{\mathbf{i}, \mathbf{m}}$ which we call the LS-path basis.

First we recall the analogous basis $\left\{v_{\pi}\right\}$ of $V_{\lambda}$ referred to in Theorem 3. Let $\widehat{s}_{i}$ be the operators of Section 2.5. Note that wt $\widehat{s}_{i}(\pi)=\mathrm{wt} \pi-l \alpha_{i}$ for $l \in \mathbb{Z}_{+}$. For an LS-chain $\pi$ of shape $\lambda$, define integers $l_{1}(\pi), l_{2}(\pi), \ldots$ by

$$
l_{1}(\pi)=\frac{\mathrm{wt} \pi-\mathrm{wt} \widehat{s}_{i_{1}}(\pi)}{\alpha_{i_{1}}}, \quad l_{2}(\pi)=\frac{\mathrm{wt} \widehat{s}_{i_{1}}(\pi)-\mathrm{wt} \widehat{s}_{i_{2}} \widehat{s}_{i_{1}}(\pi)}{\alpha_{i_{2}}}, \quad \ldots
$$

That is, wt $\pi-\mathrm{wt} \widehat{s}_{i_{1}}(\pi)=l_{1}(\pi) \alpha_{i_{1}}$, etc. Note that this depends on our fixed word $\mathbf{i}=\left(i_{1}, \ldots, i_{r}\right)$. For $\left(l_{1}, \ldots, l_{r}\right)=\left(l_{1}(\pi), \ldots, l_{r}(\pi)\right)$, define

$$
v_{\pi}^{\mathbf{i}}=F_{i_{1}}^{l_{1}} \cdots F_{i_{r}}^{l_{r}} \cdot v_{\lambda} \in V_{\lambda},
$$

where $F_{i} \in \mathbf{g}$ are negative root vectors. Now, let $w_{\max }(\mathbf{i})$ be the unique Bruhat-maximal element in the set $\{w(J) \mid J \subset[1, r]\}$; that is, $w_{\max }(\mathbf{i})$ is the Weyl group element generated by a longest reduced subword of i. (See [13, Lemma 1].) Our definition of $\left\{v_{\pi}\right\}$ is a slight generalization of [20, Definition 3], since $\mathbf{i}$ need not be reduced. Recall [20, Theorem 2]:

PROPOSITION 14. Let $w=w_{\max }(\mathbf{i})$. The set $\left\{v_{\pi}^{\mathbf{i}}\right\}$, where $\pi$ runs over all LS-chains $\pi=\left(\tau_{1}>\tau_{2}>\cdots ; 0<a_{1}<\cdots\right)$ with $w>\tau_{1}$, forms a basis of $V_{\lambda}(w)$.

We shall need one technical property of the $v_{\pi}$.

LEMMA 15. Let $\pi^{\prime}=\widehat{s}_{i}(\pi), l=\left(\mathrm{wt} \pi-\mathrm{wt} \pi^{\prime}\right) / \alpha_{i}$, and $\theta^{\prime}<\pi^{\prime}$ in the lexicographic order on LS-chains (Section 1.3). Suppose either of the following holds: (i) $k \leqslant l$; or (ii) the Raghavan-Sankaran operator coincides with ordinary reflection: 
$\pi^{\prime}=\widehat{s}_{i}(\pi)=s_{i}(\pi)$. Then

$$
F_{i}^{k}\left(v_{\theta^{\prime}}\right)= \begin{cases}v_{\pi}, & \text { if } \theta^{\prime}=\pi^{\prime} \text { and } k=l, \\ \sum_{\theta<\pi}^{\alpha v_{\theta},}, & \text { otherwise, }\end{cases}
$$

where $\theta$ runs over LS-chains less than $\pi$.

Proof. The first case of the conclusion, $F_{i}^{k}\left(v_{\theta^{\prime}}\right)=v_{\pi}$, follows directly from the definition of $v_{\pi}$. The second case of the conclusion follows easily from either hypothesis together with [20, Lemma 3(ii)].

Now we extend the above construction to generalized Demazure modules. For a tableau $\Pi$ of shape $\left(\lambda_{1}, \ldots, \lambda_{r}\right)$, we have $\widehat{s}_{i_{1}}(\Pi)=\pi^{\lambda_{1}} * \Pi^{\prime}$ for some tableau $\Pi^{\prime}$ of shape $\left(\lambda_{2}, \ldots, \lambda_{r}\right)$; and $\widehat{s}_{i_{2}}\left(\Pi^{\prime}\right)=\pi^{\lambda_{2}} * \Pi^{\prime \prime}$, etc. Then define integers

$$
L_{1}(\Pi)=\frac{\mathrm{wt} \Pi-\mathrm{wt} \widehat{s}_{i_{1}}(\Pi)}{\alpha_{i_{1}}}, \quad L_{2}(\pi)=\frac{\mathrm{wt} \Pi^{\prime}-\mathrm{wt} \widehat{s}_{i_{2}}\left(\Pi^{\prime}\right)}{\alpha_{i_{2}}}, \quad \text { etc. }
$$

(Recall that wt $\Pi$ denotes the endpoint of $\Pi$ considered as a path, i.e., the sum of the weights of the LS-chains in $\Pi$.)

Now for $\left(L_{1}, \ldots, L_{r}\right)=\left(L_{1}(\Pi), \ldots, L_{r}(\Pi)\right)$, let

$$
v_{\Pi}=F_{i_{1}}^{L_{1}}\left(v_{\lambda_{1}} \otimes F_{i_{2}}^{L_{2}}\left(v_{\lambda_{2}} \otimes \cdots F_{i_{r}}^{l_{r}}\left(v_{\lambda_{r}}\right) \cdots\right)\right) .
$$

The $\left\{v_{\Pi}\right\}$ coincide with the $\left\{v_{\pi}\right\}$ in the case where $V_{\mathbf{i}, \mathbf{m}}$ is an ordinary Demazure module $V_{\lambda}(w)$.

\subsection{INDEPENDENCE OF $\left\{p_{\pi}\right\}$}

Let $w_{j}=w_{\max }\left(i_{j}, \ldots, i_{r}\right)$, the longest Weyl group element which can be generated from a tail subword of $\mathbf{i}$. Then clearly $V_{\mathbf{i}, \mathbf{m}} \subset V_{\lambda_{1}}\left(w_{1}\right) \otimes \cdots \otimes V_{\lambda_{r}}\left(w_{r}\right)$. We will write $v_{\pi}^{(j)}=v_{\pi}^{\left(i_{j}, \ldots, i_{r}\right)}$. By Proposition 14, the vectors

$$
\left\{v_{\pi_{1}}^{(1)} \otimes \cdots \otimes v_{\pi_{r}}^{(r)}\right\}
$$

where each $\pi_{j}=\left(\tau_{1}>\cdots\right)$ varies over all LS-chains with $w_{j}>\tau_{1}$, form a basis of $V_{\lambda_{1}}\left(w_{1}\right) \otimes \cdots \otimes V_{\lambda_{r}}\left(w_{r}\right)$.

PROPOSITION 16. For a standard tableau $\Pi=\left(\pi_{1}, \ldots, \pi_{r}\right)$, let us write $v_{\Pi} \in V_{\mathbf{i}, \mathbf{m}}$ in terms of the above basis of $V_{\lambda_{1}}\left(w_{1}\right) \otimes \cdots \otimes V_{\lambda_{r}}\left(w_{r}\right)$. Then we have the triangular relation:

$$
v_{\Pi}=v_{\pi_{1}}^{(1)} \otimes \cdots \otimes v_{\pi_{r}}^{(r)}+\sum_{\Theta<\Pi} \propto v_{\theta_{1}}^{(1)} \otimes \cdots \otimes v_{\theta_{r}}^{(r)},
$$

where $\Theta=\left(\theta_{1}, \ldots, \theta_{r}\right)$ runs over all tableaux less than $\Pi$ in lexicographic order. 
Proof. (cf. [20, Proof of Lemma 3]). We use induction on $r$, the number of letters in i. For $r=1$, there is nothing to prove. Now suppose $r \geqslant 2$, and we know the Proposition for the word $\left(i_{2}, \ldots, i_{r}\right)$. Let $s=s_{i_{1}}$ and take $\Pi^{\prime}=\widehat{s}(\Pi)$, where

$$
\Pi=\left(\pi_{1}, \ldots, \pi_{r}\right), \quad \Pi^{\prime}=\left(\widehat{s}\left(\pi_{1}\right), \ldots, \widehat{s}\left(\pi_{j}\right), \pi_{j+1}, \ldots, \pi_{r}\right),
$$

and

$$
\pi^{\lambda_{1}}=\widehat{s}\left(\pi_{1}\right)=s\left(\pi_{1}\right), \widehat{s}\left(\pi_{2}\right)=s\left(\pi_{2}\right), \ldots, \widehat{s}\left(\pi_{j-1}\right)=s\left(\pi_{j-1}\right) .
$$

By induction, we may assume

$$
v_{\Pi^{\prime}}=v_{\lambda_{1}} \otimes v_{\hat{s}\left(\pi_{2}\right)} \otimes \cdots \otimes v_{\hat{s}\left(\pi_{k}\right)} \otimes \cdots \otimes v_{\pi_{r}}+\sum_{\Theta<\Pi^{\prime}} \propto v_{\lambda_{1}} \otimes v_{\theta_{2}} \otimes \cdots \otimes v_{\theta_{r}},
$$

where $\Theta=\left(\pi^{\lambda_{1}}, \theta_{2}, \ldots, \theta_{r}\right)$. Then

$$
v_{\Pi}=F_{i_{1}}^{l_{1}}\left(v_{\Pi^{\prime}}\right)
$$

where $l_{1}=h_{1}+\cdots+h_{k}$ for

$$
h_{1}=\frac{\mathrm{wt} \pi_{1}-\mathrm{wt} s\left(\pi_{1}\right)}{\alpha_{i_{1}}}, \ldots, h_{k-1}=\frac{\mathrm{wt} \pi_{k-1}-\mathrm{wt} s\left(\pi_{k-1}\right)}{\alpha_{i_{1}}}, h_{k}=\frac{\mathrm{wt} \pi_{k}-\mathrm{wt} \widehat{s}\left(\pi_{k}\right)}{\alpha_{i_{1}}} .
$$

The terms of $v_{\Pi}$ are obtained by distributing the $l_{1}$ operations $F_{i_{1}}^{l_{1}}$ arbitrarily among the $r$ factors of each term in $v_{\Pi^{\prime}}$.

We find the maximal term in $v_{\Pi}$ by repeatedly applying Lemma 15 . By hypothesis (ii) of the Lemma, the maximal first factor of a term in $v_{\Pi}$ is $F_{i_{1}}^{h_{1}}\left(v_{s\left(\pi_{1}\right)}\right)=v_{\pi_{1}}$; the maximal second factor is $v_{\pi_{2}}$; and so on through the $(k-1)$ th factor. Now, assuming the previous maximal factors have been achieved, we apply hypothesis (i) of the Lemma to find that the maximal $k$ th factor is $F_{i_{1}}^{h_{k}}\left(v_{\hat{s}\left(\pi_{k}\right)}\right)=v_{p i_{k}}$. But then all $l_{1}$ operations $F_{i_{1}}$ have been used, and the subsequent terms are unchanged from $v_{\Pi^{\prime}}$.

Recall that the set $\left\{p_{\Pi}\right\}$ inside $V_{\mathbf{i}, \mathbf{m}}^{*}$ consists of monomials in any basis $\left\{p_{\pi}\right\}$ of the $V_{\lambda}^{*}$ which is triangular with respect to $\left\{v_{\pi}\right\}$.

COROLLARY 17. (i) The set $\left\{v_{\Pi}\right\}$, where $\Pi$ runs over the standard tableaux of shape $\left(\lambda_{1}, \ldots, \lambda_{r}\right)$, is linearly independent in $V_{\mathbf{i}, \mathbf{m}}$.

(ii) The set $\left\{p_{\Pi}\right\}$, where $\Pi$ runs over the standard tableaux of shape $\left(\lambda_{1}, \ldots, \lambda_{r}\right)$, restricts to a linearly independent set in $V_{\mathbf{i}, \mathbf{m}}^{*}$.

Proof. Part (i) follows immediately from the triangularity of the set $\left\{v_{\Pi}\right\}$ with respect to the basis $\left\{v_{\pi_{1}}^{(1)} \otimes \cdots \otimes v_{\pi_{r}}^{(r)}\right\}$. Part (ii) follows similarly, using in addition the triangularity of $\left\{p_{\pi}\right\}$ with respect to $\left\{v_{\pi}\right\}$. 


\section{Spanning}

The independence of the set $\left\{p_{\Pi}\right\}$ in $V_{\mathbf{i}, \mathrm{m}}^{*}$, along with the Demazure formula for standard tableaux (Section 2.3), gives a lower bound for the dimension of $V_{\mathbf{i}, \mathbf{m}}^{*}$. In this section, we use geometry to find an upper bound for this dimension which coincides with the lower bound, showing that $\left\{p_{\Pi}\right\}$ is a basis.

\subsection{THE DEMAZURE MODULE AS A SPACE OF SECTIONS}

We relate the generalized Demazure module $V_{\mathbf{i}, \mathbf{m}}$ and the Bott-Samelson variety $Z_{\mathbf{i}}$ via a succession of three mappings (see [23]). First, let $U_{i}$ denote the one-dimensional unipotent subgroup of $G$ whose Lie algebra is $\mathbf{k} F_{i}$. We have an embedding

$$
\phi_{1}: U_{i_{1}} \times \cdots \times U_{i_{r}} \rightarrow\left(P_{i_{1}} \times \cdots \times P_{i_{r}}\right) / B^{r}=Z_{\mathbf{i}}
$$

whose image defines a Zariski-dense open cell in $Z_{\mathbf{i}}$.

Second, let $\widehat{P}_{i} \supset B$ be the maximal parabolic subgroup whose Weyl group is generated by all the simple reflections except $s_{i}$. Let $\operatorname{Gr}(i)=\operatorname{Gr}(i, G):=G / \widehat{P}_{i}$ be the $G$-Grassmannian, and define the multiple $G$-Grassmannian

$$
\operatorname{Gr}(\mathbf{i}):=\operatorname{Gr}\left(i_{1}\right) \times \cdots \times \operatorname{Gr}\left(i_{r}\right),
$$

on which $G$ acts diagonally (simultaneously on each factor). The Bott-Samelson variety embeds $B$-equivariantly into this space:

$$
\begin{array}{cccc}
\phi_{2}: & Z_{\mathbf{i}} & \hookrightarrow & \operatorname{Gr}(\mathbf{i}) \\
& \left(p_{1}, \ldots, p_{r}\right) & \mapsto
\end{array} \quad\left(p_{1} \widehat{P}_{i_{1}}, p_{1} p_{2} \widehat{P}_{i_{2}}, \ldots, p_{1} p_{2} \cdots p_{r} \widehat{P}_{i_{r}}\right) .
$$

(The configuration variety $Z_{\mathbf{i}, \mathbf{m}}$ can be realized as the projection of $Z_{\mathbf{i}} \subset \operatorname{Gr}(\mathbf{i})$ to those factors of $\operatorname{Gr}(\mathbf{i})$ for which $m_{j}>0$.)

Third, for a weight $\lambda=m \varpi_{i}$, define a line bundle on $\operatorname{Gr}(i)$ as $\mathcal{L}_{\lambda}:=G \stackrel{\widehat{P}_{i}}{\times} \mathbf{k}_{\lambda}^{*}$, so that $\mathcal{L}_{\varpi_{i}}$ is the minimal ample line bundle on $\operatorname{Gr}(i)$. We thus obtain a line bundle $\mathcal{L}_{\mathbf{i}, \mathbf{m}}:=\mathcal{L}_{\lambda_{1}} \otimes \cdots \otimes \mathcal{L}_{\lambda_{r}}$ on $\operatorname{Gr}(\mathbf{i})$, which is very ample (resp. semi-ample) precisely when all $m_{j}>0$ (resp. all $m_{j} \geqslant 0$ ). The restriction of $\mathcal{L}_{\mathbf{i}, \mathbf{m}}$ to $Z_{\mathbf{i}} \subset \operatorname{Gr}(\mathbf{i})$ is easily seen to be isomorphic to the line bundle $\mathcal{L}_{\mathbf{i}, \mathrm{m}}$ on $Z_{\mathbf{i}}$ defined in Section 1.4. Recall that we may identify $H^{0}\left(\operatorname{Gr}(i), \mathcal{L}_{\lambda}\right) \cong V_{\lambda}^{*}$, so that $\operatorname{Gr}(i) \rightarrow \mathbb{P}\left(H^{0}\left(\operatorname{Gr}(i), \mathcal{L}_{\lambda}\right)^{*}\right) \cong \mathbb{P}\left(V_{\lambda}\right)$, $g \widehat{P}_{i} \mapsto g \cdot v_{\lambda}$. Thus we have the natural map

$$
\phi_{3}: \begin{array}{ccc}
\operatorname{Gr}(\mathbf{i}) & \rightarrow & \mathbb{P}\left(V_{\lambda_{1}} \otimes \cdots \otimes V_{\lambda_{r}}\right) \\
\left(g_{1} \widehat{P}_{i_{1}}, \ldots, g_{r} \widehat{P}_{i_{r}}\right) & \mapsto & g_{1} \cdot v_{\lambda_{1}} \otimes \ldots \otimes g_{r^{\prime}} v_{\lambda_{r}} .
\end{array}
$$

Now, composing $\phi_{3} \circ \phi_{2}$, we have a map

$$
Z_{\mathbf{i}} \rightarrow \mathbb{P}\left(V_{\lambda_{1}} \otimes \cdots \otimes V_{\lambda_{r}}\right)
$$

whose image is by definition $Z_{\mathbf{i}, \mathbf{m}}$. If we compose all three mappings $\phi_{3} \circ \phi_{2} \circ \phi_{1}$, we 
see that:

$$
\begin{aligned}
\mathbb{P}\left(V_{\lambda_{1}} \otimes \cdots \otimes V_{\lambda_{r}}\right) & \supset \operatorname{Span}_{\mathbf{k}}\left\langle Z_{\mathbf{i}, \mathbf{m}}\right\rangle \\
& =\operatorname{Span}_{\mathbf{k}}\left\langle U_{i_{1}}\left(v_{\lambda_{1}} \otimes U_{i_{2}}\left(v_{\lambda_{2}} \otimes \cdots U_{i_{r}} v_{\lambda_{r}} \cdots\right)\right)\right\rangle \\
& =\mathbb{P}\left(\mathbf{u}_{i_{1}}\left(v_{\lambda_{1}} \otimes \mathbf{u}_{i_{2}}\left(v_{\lambda_{2}} \otimes \cdots \mathbf{u}_{i_{r}} v_{\lambda_{r}} \cdots\right)\right)\right) \\
& =\mathbb{P}\left(V_{\mathbf{i}, \mathbf{m}}\right)
\end{aligned}
$$

That is, we have the map $\phi=\phi_{3} \circ \phi_{2}: Z_{\mathbf{i}} \rightarrow \mathbb{P}\left(V_{\mathbf{i}, \mathbf{m}}\right)$ whose image spans $\mathbb{P}\left(V_{\mathbf{i}, \mathbf{m}}\right)$. Dually, we have the injective linear map $\phi^{*}$, which factors as:

$$
V_{\mathbf{i}, \mathbf{m}}^{*} \hookrightarrow H^{0}\left(Z_{\mathbf{i}, \mathbf{m}}, \mathcal{L}_{\mathbf{i}, \mathbf{m}}\right) \hookrightarrow H^{0}\left(Z_{\mathbf{i}}, \mathcal{L}_{\mathbf{i}, \mathbf{m}}\right) .
$$

\subsection{GEOMETRIC DEMAZURE FORMULA}

We now use Demazure's character computations with $\mathbb{P}^{1}$-fibrations [3] to finish our proof of Theorems 4, 5, and 6, on the model of [20, § 8].

Our proof proceeds by induction on $r$, the number of letters in i. For $r=0$, all statements are trivial. Now let $r \geqslant 1, i=i_{1}$, and $\mathbf{i}^{\prime}=\left(i_{2}, \ldots, i_{r}\right), \mathbf{m}^{\prime}=\left(m_{2}, \ldots, m_{r}\right)$.

From the definitions, we easily see that $H^{0}\left(Z_{\mathbf{i}}, \mathcal{L}_{\mathbf{i}, \mathbf{m}}\right)=H^{0}\left(P_{i} / B, \mathcal{E}\right)$, where

$$
\mathcal{E}=P_{i} \stackrel{B}{\times}\left(\mathbf{k}_{\lambda_{1}}^{*} \otimes H^{0}\left(Z_{\mathbf{i}^{\prime}}, \mathcal{L}_{\mathbf{i}^{\prime}, \mathbf{m}^{\prime}}\right)\right)=P_{i} \stackrel{B}{\times}\left(\mathbf{k}_{\lambda_{1}}^{*} \otimes V_{\mathbf{i}^{\prime}, \mathbf{m}^{\prime}}^{*}\right),
$$

a vector bundle over $P_{i} / B$. (The last equality is by induction.) Now restrict the $P_{i}$-action on this vector bundle to an action of $G_{i} \cong S L_{2}$, the group whose Lie algebra is generated by $E_{i}, F_{i}$. Take $B_{i}=B \cap G_{i}$, so that $P_{i} / B \cong G_{i} / B_{i} \cong \mathbb{P}^{1}$.

The inclusion $v_{\lambda_{1}} \otimes V_{\mathbf{i}^{\prime}, \mathbf{m}^{\prime}} \subset V_{\mathrm{i}, \mathbf{m}}$ dualizes to a short exact sequence of $B_{i}$-modules $0 \rightarrow \operatorname{Ker} \rightarrow V_{\mathbf{i}, \mathbf{m}}^{*} \rightarrow \mathbf{k}_{\lambda_{1}}^{*} \otimes V_{\mathbf{i}^{\prime}, \mathbf{m}^{\prime}}^{*} \rightarrow 0$, which leads to a long exact sequence in cohomology of bundles over $\mathbb{P}^{1}$ :

$$
\cdots \rightarrow H^{i}\left(\mathbb{P}^{1}, G_{i} \times B_{i} \mathrm{Ker}\right) \rightarrow H^{i}\left(\mathbb{P}^{1}, G_{i} \times{ }_{i}^{B_{i}} V_{\mathrm{i}, \mathrm{m}}\right) \rightarrow H^{i}\left(\mathbb{P}^{1}, \mathcal{E}\right) \rightarrow \cdots
$$

Since $V_{\mathbf{i}, \mathbf{m}}$ is a $G_{i}$-module, it induces a trivial vector bundle, and $H^{1}\left(\mathbb{P}^{1}\right.$, $\left.G_{i} \stackrel{B_{i}}{\times} V_{\mathbf{i}, \mathbf{m}}\right)=0$. Since trivially $H^{2}\left(\mathbb{P}^{1}, G_{i} \times B_{i} \times\right.$ Ker $)=0$, we thus get:

$$
H^{i}\left(\mathbb{P}^{1}, \mathcal{E}\right)=0 \text { for } i>0 .
$$

From the Leray spectral sequence of the fibration $Z_{\mathbf{i}} \rightarrow P_{i} / B$, and induction, it follows that $H^{i}\left(Z_{\mathbf{i}}, \mathcal{L}_{\mathbf{i}, \mathbf{m}}\right)=0$ for $i>0$.

Now, the character ring of $G_{i}$ is $R_{i} \cong \mathbf{k}[x]$, a polynomial ring in one variable, with the quotient map $R \rightarrow R_{i}$, $\mathrm{e}^{\lambda} \mapsto x^{\left\langle\lambda, \alpha_{i}^{\vee}\right\rangle}$. From elementary computations with $S L_{2}$-bundles we have Demazure's formula [3] for the $G_{i}$-character char ${ }_{i}$ of the cohomology of $\mathcal{E}$, in terms of the fiber of $\mathcal{E}$ above $e B_{i} \in G_{i} / B_{i}$ :

$$
\operatorname{char}_{i} H^{0}\left(\mathbb{P}^{1}, \mathcal{E}\right)-\operatorname{char}_{i} H^{1}\left(\mathbb{P}^{1}, \mathcal{E}\right)=\Lambda_{i} \operatorname{char}_{i}\left(\left.\mathcal{E}\right|_{e B_{i}}\right),
$$

where $\Lambda_{i}: R_{i} \rightarrow R_{i}$ is the map induced from $\Lambda_{i}: R \rightarrow R$. But the negative $H^{1}$ term 
vanishes, and by specializing the polynomials to $x=1$ we find

$$
\begin{aligned}
\operatorname{dim} H^{0}\left(Z_{\mathbf{i}}, \mathcal{L}_{\mathbf{i}, \mathbf{m}}\right) & =\operatorname{dim} H^{0}\left(\mathbb{P}^{1}, \mathcal{E}\right) \\
& =\left.\Lambda_{i} \operatorname{char}_{i}\left(\mathbf{k}_{\lambda_{1}}^{*} \otimes V_{\mathbf{i}^{\prime}, \mathbf{m}^{\prime}}\right)\right|_{x=1} \\
& =\left.\Lambda_{i_{1}}\left(\mathrm{e}^{\lambda_{1}} \Lambda_{i_{2}}\left(\mathrm{e}^{\lambda_{2}} \cdots\right)\right)\right|_{\mathrm{e}^{\lambda}=1}
\end{aligned}
$$

where the last equality is by induction. However, we also know:

$$
\begin{aligned}
\operatorname{dim} H^{0}\left(Z_{\mathbf{i}}, \mathcal{L}_{\mathbf{i}, \mathbf{m}}\right) & \geqslant \operatorname{dim} H^{0}\left(Z_{\mathbf{i}, \mathbf{m}}, \mathcal{L}_{\mathbf{i}, \mathbf{m}}\right) \quad \text { by Section } 4.2 \\
& \geqslant \operatorname{dim} V_{\mathbf{i}, \mathbf{m}}^{*} \quad \text { by Section } 4.2 \\
& \left.\geqslant \operatorname{dim} \operatorname{Span}\left\langle p_{\Pi}\right| \Pi \text { a standard tableau }\right\rangle \\
& =\#\{\text { standard tableaux }\} \quad \text { by Corollary } 17 \\
& =\left.\Lambda_{i_{1}}\left(\mathrm{e}^{\lambda_{1}} \Lambda_{i_{2}}\left(\mathrm{e}^{\lambda_{2}} \ldots\right)\right)\right|_{\mathrm{e}^{\lambda}=1} \quad \text { by Corollary } 10
\end{aligned}
$$

Comparing expressions, we conclude that all the above inequalities are in fact equalities, meaning

$$
H^{0}\left(Z_{\mathbf{i}}, \mathcal{L}_{\mathbf{i}, \mathbf{m}}\right) \cong H^{0}\left(Z_{\mathbf{i}, \mathbf{m}}, \mathcal{L}_{\mathbf{i}, \mathbf{m}}\right) \cong V_{\mathbf{i}, \mathbf{m}}^{*}=\operatorname{Span}\left\langle p_{\Pi}\right\rangle,
$$

and the Demazure character formula holds for all four of these spaces. This implies the projective normality of $Z_{\mathbf{i}}$ and $Z_{\mathbf{i}, \mathbf{m}}$ with respect to $\mathcal{L}_{\mathbf{i}, \mathbf{m}}$ by [4, Ch II, Ex 5.14].

Finally, the vanishing of the higher cohomology of $\mathcal{L}_{\mathbf{i}, \mathbf{m}}$ over $Z_{\mathbf{i}, \mathbf{m}}$ follows from a standard argument involving the map $\phi: Z_{\mathbf{i}} \rightarrow Z_{\mathbf{i}, \mathbf{m}}$ (see, e.g. [20, § 8], [22, Proposition 28]). Using $H^{0}\left(Z_{\mathbf{i}, \mathbf{m}}, \mathcal{L}_{\mathbf{i}, \mathbf{m}}\right)=H^{0}\left(Z_{\mathbf{i}}, \mathcal{L}_{\mathbf{i}, \mathbf{m}}\right)$, and the normality of $Z_{\mathbf{i}, \mathbf{m}}$, we apply Kempf's Lemma [9] to deduce $H^{i}\left(Z_{\mathbf{i}, \mathbf{m}}, \mathcal{L}_{\mathbf{i}, \mathbf{m}}\right)=H^{i}\left(Z_{\mathbf{i}}, \mathcal{L}_{\mathbf{i}, \mathbf{m}}\right)$ for all $i>0$. But we have already shown that the right hand side vanishes.

This completes the proof of Theorems 4, 5, and 6.

\section{References}

1. Bott, R. and Samelson, H.: Applications of the theory of Morse to symmetric spaces, J. Differential Geom 1 (1967), 311-330.

2. De Concini, C.: Symplectic standard tableaux, Adv. in Math. 34 (1979), 1-27.

3. Demazure, M.: Désingularisation des variétés de Schubert généralisées, Ann. Sci. Ecole Norm. Sup. 7 (1974), 53-88.

4. Hartshorne, R.: Algebraic Geometry, Grad. Texts Math. 52, Springer, New York, 1977.

5. Humphreys, J. E.: Reflection Groups and Coxeter Groups, Cambridge Univ. Press, 1990.

6. Joseph, A.: Quantum Groups and their Primitive Ideals, Springer, New York, 1991.

7. Kashiwara, M.: Crystal base and Littelmann's refined Demazure character formula, Duke Math. J. 71 (1993), 839-858.

8. Kashiwara, M.: On Crystal bases of the $q$-analog of universal enveloping algebras, Duke Math. J. 63 (1991), 465-516.

9. Kempf, G.: Linear systems on homogeneous spaces, Ann. Math. 103 (1976), 557-591.

10. Kumar, S.: Demazure character formula in arbitrary Kac-Moody setting, Invent. Math. 89 (1987), 395-423. 
11. Lakshmibai, V.: Bases for quantum Demazure modules, In: B. N. Allison and G. H. Cliff (eds) Representations of Groups, CMS Conf. Proc. 16, Am. Math. Soc., Providence, 1995.

12. Lakshmibai, V. and Magyar, P.: Standard monomial theory for Bott-Samelson varieties, C.R. Acad. Sci. Paris Ser I 324 (1997), 1211-1215.

13. Lakshmibai, V. and Magyar, P.: Standard monomial theory for Bott-Samelson varieties of GL(n), Publ. RIMS Kyoto 34 (1998), 229-248.

14. Lakshmibai, V. and Seshadri, C. S.: Geometry of G/P-IV, Proc. Indian Acad. Sci. A 88 (1979), 279-362.

15. Lakshmibai, V. and Seshadri, C. S.: Geometry of $G / P-V$, J. Algebra 100 (1986), $462-557$.

16. Lakshmibai, V. and Seshadri, C. S.: Standard monomial theory, In: S. Ramanan (ed.) Proceedings Hyderabad Conference on Algebraic Groups, Manoj Prakashan Publishers, Madras, 1991.

17. Littelmann, P.: A Littlewood-Richardson rule for symmetrizable Kac-Moody algebras, Invent. Math. 116 (1994), 329-346.

18. Littelmann, P.: Paths and root operators in representation theory, Ann. of Math. 142 (1995), 499-525.

19. Littelmann, P.: A plactic algebra for semisimple Lie algebras, Adv. Math. 124 (1996), 312-331.

20. Littelmann, P.: Contracting modules and standard monomial theory for symmetrizable Kac-Moody algebras, J. Amer. Math. Soc. 11 (1998), 551-567.

21. Lusztig, G.: Introduction to Quantum Groups, Progr. Math. 110, Birkhauser Basel, 1993.

22. Magyar, P.: Borel-Weil theorem for configuration varieties and Schur modules, $A d v$. Math. 134 (1998), 328-366.

23. Magyar, P.: Schubert polynomials and Bott-Samelson varieties, Comment. Math. Helv. 73 (1998), 603-636.

24. Marsh, R.: Algorithms to obtain the canonical basis in some fundamental modules of quantum groups, J. Algebra 186 (1996), 831-860.

25. Mathieu, O.: Filtrations of B-modules, Duke Math. J. 59 (1989), 421-442.

26. Raghavan, K. N. and Sankaran, P. A.: A new approach to standard monomial theory for classical groups, Transform. Groups 3 (1998), 57-73.

27. Van der Kallen, W.: Frobenius Splittings and B-modules, Tata Inst. Res. Lecture 84, Springer, New York, 1993. 